\title{
Prediction of Structures and Interactions from Genome Information
}

\author{
Miyazawa, Sanzo
}

\begin{abstract}
Predicting three dimensional residue-residue contacts from evolutionary information in protein sequences was attempted already in the early 1990s. However, contact prediction accuracies of methods evaluated in CASP experiments before CASP11 remained quite low, typically with $<20 \%$ true positives. Recently, contact prediction has been significantly improved to the level that an accurate three dimensional model of a large protein can be generated on the basis of predicted contacts. This improvement was attained by disentangling direct from indirect correlations in amino acid covariations or cosubstitutions between sites in protein evolution. Here, we review statistical methods for extracting causative correlations and various approaches to describe protein structure, complex, and flexibility based on predicted contacts.
\end{abstract}

Keywords: contact prediction; direct coupling; amino acid covariation; amino acid cosubstitution; partial correlation; maximum entropy model; inverse Potts model; Markov random field; Boltzmann machine; deep neural network

\section{Introduction}

The evolutionary history of protein sequences is a valuable source of information in many fields of science not only in evolutionary biology but even to understand protein structures. Residue-residue interactions that fold a protein into a unique threedimensional (3D) structure and make it play a specific function impose structural and functional constraints in varying degrees on each amino acid. Selective constraints on amino acids are recorded in amino acid orders in homologous protein sequences and also in the evolutionary trace of amino acid substitutions. Negative effects caused by mutations at one site must be compensated by successive muta-

Sanzo Miyazawa, e-mail: sanzo.miyazawa@gmail.com 
tions at other sites [109, 28, 56], causing covariations/cosubstitutions/coevolution between sites [104, 29, 21, 20], otherwise most negative mutants will be eliminated from a gene pool and never reach fixation in population. Such structural and functional constraints arise from interactions between sites mostly in close spatial proximity. Thus, it has been suggested and also shown that the types of amino acids [55, 53, 54, 87, 93, 11, 106, 35, 12, 71, 57] and amino acid substitutions $[3,33,92,80,81,5,25,30,29,21,59,24,17,20,18,82,19,34]$ are correlated between sites that are close in a protein 3D structure. However, until CASP11, contact prediction accuracy remained quite low, typically with $\leq 20 \%$ true positives for top- $L / 5$ long-range contacts in free modeling targets [52]; $L$ denotes protein length. Recently contact prediction has been significantly improved to the level that an accurate three dimensional model of a large protein $(\simeq 250$ residues) can be generated on the basis of predicted contacts[73]. These improvements were attained primarily by disentangling direct from indirect correlations in amino acid covariations or cosubstitutions between sites in protein evolution, and secondarily by reducing phylogenetic biases in a multiple sequence alignment (MSA) or removing them on the basis of a phylogenetic tree; see Fig. 1 .

Here, we review statistical methods for extracting causative correlations in amino acid covariations/cosubstitutions between sites, and various approaches to describe protein structure, complex and flexibility based on predicted contacts. Mathematical formulation of each statistical method is concisely described in the unified manner in an appendix. This manuscript was published in [69] with the short version of the appendix.

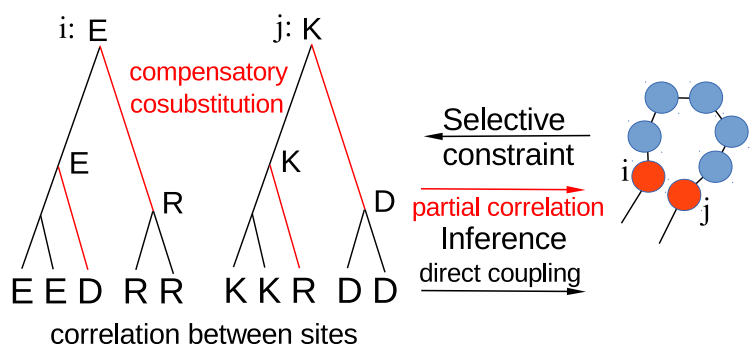

Fig. 1 Amino acids at sites $i$ and $j$ in a MSA are shown with a phylogenetic tree. Causative correlations between sites in protein evolution are extracted from the MSA or phylogenetic tree, and utilized to infer close residue pairs.

\section{Statistical methods to extract causative interactions between sites}

The primary task to develop a robust method toward contact prediction is to detect causative correlations, which reflect evolutionary constraints, in amino acid covaria- 
tions between sites in a multiple sequence alignment (MSA) or in amino acid cosubstitutions between sites in branches of a phylogenetic tree; see Table 1 . The former was called direct coupling analysis (DCA)[71].

Table 1 Statistical methods for disentangling direct from indirect correlations between sites.

\begin{tabular}{|c|c|}
\hline \multicolumn{2}{|l|}{ Category } \\
\hline Method name & Method/algorithm \\
\hline \multicolumn{2}{|c|}{ A) Direct coupling analysis of amino acid covariations between sites in a MSA } \\
\hline Boltzmann machine & $\begin{array}{l}\text { Markov chain Monte Carlo to calculate marginal probabilities and } \\
\text { gradient descent to estimate fields and couplings. }\end{array}$ \\
\hline CMI [54] & Boltzmann machine to estimate conditional mutual information \\
\hline mpDCA [106] & $\begin{array}{l}\text { Message-passing algorithm to estimate marginal probabilities } \\
\text { and gradient descent to estimate fields and couplings }\end{array}$ \\
\hline mfDCA 71 57] & Mean field approximation to estimate the partition function \\
\hline PSICOV [45] & $\begin{array}{l}\text { Graphical lasso (Gaussian approximation with an exponential prior) } \\
\text { with a shrinkage method for a covariance matrix }\end{array}$ \\
\hline GaussDCA [7] & A multivariate Gaussian model with a normal-inverse-Wishart prior \\
\hline plmDCA 23 22] & Pseudo-likelihood maximization with Gaussian priors ( $\ell_{2}$ regularizers) \\
\hline GREMLIN 6, 48] & $\begin{array}{l}\text { Pseudo-likelihood maximization with } \ell_{1} \text { regularization terms } 6 \text { ] which depend on site pair } \\
\text { or with Gaussian priors } 48 \text { w }\end{array}$ \\
\hline ACE $15,16 \quad 8$ & Adaptive cluster expansion of cross-entropy with Gaussian priors \\
\hline Persistent VI \& Fadeout & Variational inference with sparsity-inducing prior, horseshoe [40] \\
\hline 98] & Boltzmann machine with $\ell_{2}$ regularization terms \\
\hline DI 101 & Partial correlation of normalized mutual informations between sites \\
\hline \multicolumn{2}{|c|}{ B) Partial correlation analysis of amino acid cosubstitutions between sites in a phylogenetic tree } \\
\hline $\mathrm{pcSV} 66$ & $\begin{array}{l}\text { Partial correlation coefficients of coevolutionary substitutions } \\
\text { between sites within branches in a phylogenetic tree }\end{array}$ \\
\hline
\end{tabular}

\subsection{Direct coupling analysis for amino acid covariations between sites in a multiple sequence alignment}

The direct coupling analysis is based on the maximum entropy model for the distribution of protein sequences, which satisfies the observed statistics in a MSA.

\subsubsection{Maximum entropy model for the distribution of protein sequences}

Let us consider probability distributions $P(\sigma)$ of amino acid sequences, $\sigma \equiv\left(\sigma_{1}, \ldots, \sigma_{L}\right)^{T}$ with $\sigma_{i} \in$ \{amino acids, deletion\}, single-site and two-site marginal probabilities of which are equal to a given frequency $P_{i}\left(a_{k}\right)$ of amino acid $a_{k}$ at each site $i$ and a given frequency $P_{i j}\left(a_{k}, a_{l}\right)$ of amino acid pair $\left(a_{k}, a_{l}\right)$ for site pair $(i, j)$, respectively. 


$$
\begin{gathered}
P\left(\sigma_{i}=a_{k}\right) \equiv \sum_{\sigma} P(\sigma) \delta_{\sigma_{i} a_{k}}=P_{i}\left(a_{k}\right) \\
P\left(\sigma_{i}=a_{k}, \sigma_{j}=a_{l}\right) \equiv \sum_{\sigma} P(\sigma) \delta_{\sigma_{i} a_{k}} \delta_{\sigma_{j} a_{l}}=P_{i j}\left(a_{k}, a_{l}\right)
\end{gathered}
$$

where $a_{k} \in\{$ amino acids, deletion $\}, k=1, \ldots, q, q \equiv \mid\{$ amino acids, deletion $\} \mid=21$, $i, j=1, \ldots, L$, and $\delta_{\sigma_{i} a_{k}}$ is the Kronecker delta. The distribution $P_{\mathrm{ME}}$ with the maximum entropy is

$$
\begin{aligned}
& P_{\mathrm{ME}}(\sigma \mid h, J) \\
& =\arg \max _{P(\sigma)}\left[-\sum_{\sigma} P(\sigma) \log P(\sigma)+\lambda\left(\sum_{\sigma} P(\sigma)-1\right)\right. \\
& \quad+\sum_{i}\left[h_{i}\left(a_{k}\right)\left(\sum_{\sigma} P(\sigma) \delta_{\sigma_{i} a_{k}}-P_{i}\left(a_{k}\right)\right)\right] \\
& \left.\quad+\sum_{i} \sum_{j>i}\left[J_{i j}\left(a_{k}, a_{l}\right)\left(\sum_{\sigma} P(\sigma) \delta_{\sigma_{i} a_{k}} \delta_{\sigma_{j} a_{l}}-P_{i j}\left(a_{k}, a_{l}\right)\right)\right]\right]=\frac{1}{Z} e^{-H_{\mathrm{Potts}}(\sigma \mid h, J)}
\end{aligned}
$$

where $\lambda, h_{i}\left(a_{k}\right)$, and $J_{i j}\left(a_{k}, a_{l}\right)$ are Lagrange multipliers, and a Hamiltonian $H_{\text {Potts }}$, which is called that of the Potts model for $q>2$ (or the Ising model for $q=2$ ), and a partition function $Z$ are defined as

$$
-H_{\text {Potts }}(\sigma \mid h, J)=\sum_{i} h_{i}\left(\sigma_{i}\right)+\sum_{i<j} J_{i j}\left(\sigma_{i}, \sigma_{j}\right) \quad, \quad Z=\sum_{\sigma} e^{-H_{\text {Potts }}(\sigma \mid h, J)}
$$

where $h_{i}\left(a_{k}\right)$ and $J_{i j}\left(a_{k}, a_{l}\right)$ are interaction potentials called fields and couplings.

Although pairwise frequencies $P_{i j}\left(a_{k}, a_{l}\right)$ reflect not only direct but indirect correlations in amino acid covariations between sites, couplings $J_{i j}\left(a_{k}, a_{l}\right)$ reflect causative correlations only. Thus, it is essential to estimate fields and couplings from marginal probabilities. This model is called the inverse Potts model.

\subsubsection{Log-likelihood and log-posterior-probability}

Log-posterior-probability and log-likelihood for the Potts model are

$$
\begin{aligned}
\log P_{\text {post }}(h, J \mid\{\sigma\}) & \propto \ell_{\text {Potts }}\left(\left\{P_{i}\right\},\left\{P_{i j}\right\} \mid h, J\right)+\log P_{0}(h, J) \\
\ell_{\text {Potts }}\left(\left\{P_{i}\right\},\left\{P_{i j}\right\} \mid h, J\right) & =B \sum_{\sigma} P_{\text {obs }}(\sigma) \log P_{\mathrm{ME}}(\sigma \mid h, J)
\end{aligned}
$$

where $P_{\mathrm{obs}}\left(\equiv \sum_{\tau=1}^{B} \delta_{\sigma \sigma^{\tau}} / B\right)$ is the observed distribution of $\sigma$ specified with $\left\{P_{i}\left(a_{k}\right)\right\}$ and $\left\{P_{i j}\left(a_{k}, a_{l}\right)\right\}$, and $B$ is the number of instances; sequences $\sigma^{\tau}$ are assumed here to be independently and identically distributed samples in sequence space. $P_{0}(h, J)$ is a prior probability of $(h, J)$.

Let us define cross entropy[16] as the negative log-posterior-probability per instance. 


$$
\begin{aligned}
S_{0}\left(h, J \mid\left\{P_{i}\right\},\left\{P_{i j}\right\}\right) & \propto-\left(\log P_{\text {post }}(h, J \mid\{\sigma\})\right) / B \\
& \equiv S_{\text {Potts }}\left(h, J \mid\left\{P_{i}\right\},\left\{P_{i j}\right\}\right)+R(h, J)
\end{aligned}
$$

where the cross entropy $S_{\text {Potts }}$, which is the negative log-likelihood per instance for the Potts model, and the negative log-prior per instance $R$ are defined as follows.

$$
\begin{aligned}
& S_{\text {Potts }}\left(h, J \mid\left\{P_{i}\right\},\left\{P_{i j}\right\}\right) \equiv-\ell_{\text {Potts }}\left(\left\{P_{i}\right\},\left\{P_{i j}\right\} \mid h, J\right) / B \\
& \quad=\log Z(h, J)-\sum_{i} \sum_{k} h_{i}\left(a_{k}\right) P_{i}\left(a_{k}\right)-\sum_{i} \sum_{k} \sum_{j>i} \sum_{l} J_{i j}\left(a_{k}, a_{l}\right) P_{i j}\left(a_{k}, a_{l}\right) \\
& R(h, J) \equiv-\log \left(P_{0}(h, J)\right) / B
\end{aligned}
$$

The maximum likelihood estimates of $h$ and $J$, which minimize the cross entropy with $R=0$, satisfy the following equations.

$$
\frac{\partial \log Z(h, J)}{\partial h_{i}\left(a_{k}\right)}=P_{i}\left(a_{k}\right) \quad, \quad \frac{\partial \log Z(h, J)}{\partial J_{i j}\left(a_{k}, a_{l}\right)}=P_{i j}\left(a_{k}, a_{l}\right)
$$

It is, however, hardly tractable to computationally evaluate the partition function $Z(h, J)$ for any reasonable system size as a function of $h$ and $J$. Thus, approximate maximization of the log-likelihood or minimization of the cross entropy is needed to estimate $h$ and $J$.

The minimum of the cross entropy with $R=0$ for the Potts model is just the Legendre transform of $\log Z(h, J)$ from $(h, J)$ to $\left(\left\{P_{i}\right\},\left\{P_{i j}\right\}\right)$, (Eq. 10$)$, and is equal to the entropy of the Potts model satisfying Eqs. 1 and 2 ,

$$
S_{\text {Potts }}\left(\left\{P_{i}\right\},\left\{P_{i j}\right\}\right) \equiv \min _{h, J} S_{\text {Potts }}\left(h, J \mid\left\{P_{i}\right\},\left\{P_{i j}\right\}\right)=\sum_{\sigma}-P(\sigma) \log P(\sigma)
$$

The cross entropy $S_{\text {Potts }}\left(h, J \mid\left\{P_{i}\right\},\left\{P_{i j}\right\}\right)$ in Eq. 10 is invariant under a certain transformation of fields and couplings, $J_{i j}\left(a_{k}, a_{l}\right) \rightarrow J_{i j}\left(a_{k}, a_{l}\right)-J_{i j}^{1}\left(a_{k}\right)-J_{j i}^{1}\left(a_{l}\right)+J_{i j}^{0}$, $h_{i}\left(a_{k}\right) \rightarrow h_{i}\left(a_{k}\right)-h_{i}^{0}+\sum_{j \neq i} J_{i j}^{1}\left(a_{k}\right)$ for any $J_{i j}^{1}\left(a_{k}\right), J_{i j}^{0}$ and $h_{i}^{0}$. This gauge-invariance reduces the number of independent variables in the Potts model to $(q-1) L$ fields and $(q-1) L \times(q-1) L$ couplings.

A prior $P_{0}(h, J)$ yields regularization terms for $h$ and $J[16]$. If a Gaussian distribution is employed for the prior, then it will yield $\ell_{2}$ norm regularization terms. $\ell_{1}$ norm regularization corresponds to the case of exponential priors. Given marginal probabilities, the estimates of fields and couplings are those minimizing the cross entropy.

$$
(h, J)=\arg \min _{(h, J)} S_{0}\left(h, J \mid\left\{P_{i}\right\},\left\{P_{i j}\right\}\right), S_{0}\left(\left\{P_{i}\right\},\left\{P_{i j}\right\}\right) \equiv \min _{(h, J)} S_{0}\left(h, J \mid\left\{P_{i}\right\},\left\{P_{i j}\right\}\right)
$$

Since $S_{0}\left(\left\{P_{i}\right\},\left\{P_{i j}\right\}\right)$ is the Legendre transform of $(\log Z(h, j)+R(h, J))$ from $(h, J)$ to $\left(\left\{P_{i}\right\},\left\{P_{i j}\right\}\right)$, these optimum $h$ and $J$ can also be calculated from

$$
h_{i}\left(a_{k}\right)=-\frac{\partial S_{0}\left(\left\{P_{i}\right\},\left\{P_{i j}\right\}\right)}{\partial P_{i}\left(a_{k}\right)} \quad, \quad J_{i j}\left(a_{k}, a_{l}\right)=-\frac{\partial S_{0}\left(\left\{P_{i}\right\},\left\{P_{i j}\right\}\right)}{\partial P_{i j}\left(a_{k}, a_{l}\right)}
$$


In most methods for contact prediction, residue pairs are predicted as contacts in the decreasing order of score $\left(\mathcal{S}_{i j}\right)$ calculated from fields $\left\{J_{i j}\left(a_{k}, a_{l}\right) \mid 1 \leq k, l<q\right\}$; see Eqs. 80 and 83

\subsubsection{Inverse Potts model}

The problem of inferring interactions from observations of instances has been studied as inverse statistical mechanics, particularly inverse Potts model for Eq. 4 , in the filed of statistical physics, as a Markov random field, Markov network or undirected graphical model in the domain of physics, statistics and information science, and as Boltzmann machine in the field of machine learning.

The maximum-entropy approach to the prediction of residue-residue contacts toward protein structure prediction from residue covariation patterns was first described in 2002 by Lapedes and collaborators [32, 55, 53, 54]. They estimated conditional mutual information (CMI) defined in Eq. 79, which was employed as a score for residue-residue contacts, for each site pair by Boltzmann leaning with Monte Carlo importance sampling to calculate equilibrium averages and gradient descent to minimize the cross entropy and successfully predicted contacts for 11 small proteins.

Calculating marginal probabilities for given fields and couplings by Monte Carlo simulations in Boltzmann machine is very computationally intensive. To reduce a computational load, the message passing algorithm, which is exact for a tree topology of couplings but approximate for the present model, is employed instead in mpDCA[106]. Because even the message passing algorithm is too slow to be applied to a large-scale analysis across many protein families, the mean field approximation is employed in mfDCA[71, 57]; see Eq. 42, $J^{M F}=-C^{-1}$, where $C_{i j}\left(a_{k}, a_{l}\right) \equiv P_{i j}\left(a_{k}, a_{l}\right)-P_{i}\left(a_{k}\right) P_{j}\left(a_{l}\right)$. In the mean field approximation, a bottleneck in computation is the calculation of the inverse of a covariance matrix $C$ that is a $(q-1) L \times(q-1) L$ matrix defined in Eq. 39 In the mean field approximation, a prior distribution in Eq. 11 is ignored and pseudocount is employed instead of regularization terms to make the covariance matrix invertible.

The Gaussian approximation (a continuous multivariate Gaussian model) for the probability distribution of sequences is employed together with an exponential prior (an $\ell_{1}$ regularization term) in PSICOV [45], and with a normal-inverseWishart (NIW) prior, which is a conjugate distribution of the multivariate Gaussian, in GaussDCA[7]. The use of NIW prior has a merit that fields and couplings can be analytically formulated; see Eqs. 62. and 63.

All methods based on the Gaussian approximation employ the analytical formula for couplings, $J \simeq-C^{-1}=-\Theta$, which are essentially as same as the mean field approximation with a difference that the covariance matrix $(C)$ or precision matrix $(\Theta)$ is differently estimated based on the various priors. The mean field and Gaussian approximations may be appropriate to systems of dense and weak couplings but questionable for sparse and strong couplings that is the characteristic of residueresidue contact networks. Although the mean field and Gaussian approximations 
successfully predict residue-residue contacts in proteins, it has been shown[8, 14] that they do not give the accurate estimates of fields and couplings in proteins.

A pseudo-likelihood with Gaussian priors ( $\ell_{2}$ regularization terms) is maximized to estimate fields and couplings in plmDCA[23, 22] for the Potts model with sparse interactions as well as reducing computational time; see Eq. 70 for the symmetric plmDCA and Eq. 73 for the asymmetric plmDCA. The asymmetric plmDCA method[22] requires less computational time and fits particularly with parallel computing.

GREMLIN[48] employs together with pseudo-likelihood Gaussian priors that depend on site pair, although its earlier version[6] employed $\ell_{1}$ regularizers, which may be more appropriate to systems of sparse couplings. The $\ell_{1}$ regularizers appear to learn parameters that are closer to their true strength, but the $\ell_{2}$ regularizers appear to be as good as the $\ell_{1}$ regularizers for the task of contact prediction that requires the relative ranking of the interactions and not their actual values [48].

One of approaches to surpass the pseudo-likelihood approximation for systems of sparse couplings may be the adaptive cluster expansion (ACE) of cross entropy [15, 16, 8], in which cross entropy is approximately minimized by taking account of only site clusters the incremental entropy (cluster entropy) of which by adding one more site is significant. In this method[8], a Boltzmann machine is employed to refine fields and couplings and also to calculate model correlations such as single-site and pairwise amino acid frequencies under given fields and couplings. The results of the Boltzmann machine for both biological and artificial models showed that ACE outperforms plmDCA in recovering single-site marginals (amino acid frequencies at each site) and the distribution of the total dimensionless energies $\left(H_{\text {Potts }}(\sigma)\right)$ [8]; those models were a lattice protein, trypsin inhibitor, HIV p7 nucleocapsid protein, multi-electrode recording of cortical neurons, and Potts models on Eridös-Rényi random graphs. More importantly ACE could accurately recover the true fields $h$ and couplings $J$ corresponding to Potts states with $P_{i}\left(a_{k}\right) \geq 0.05$ for Potts models $(L=50)$ on Eridös-Rényi random graphs[8]. On the other hand, plmDCA gave accurate estimates of couplings at weak regularization for well sampled single-site probabilities, but less accurate fields. Also, plmDCA yielded less well inferred fields and couplings for single-site and two-site probabilities not well sampled, indicating that not well populated states should be merged. As a result, the distribution of the total energies [8] and the distribution of mutations with respect to the consensus sequence were not well reproduced[14]. Similarly, the mean field approximation could not reproduce two-site marginals and even single-site marginals [14] and the Gaussian approximation could not well reproduce the distribution of mutations with respect to the consensus sequence [8].

However, the less reproducibility of couplings does not necessarily indicate the less predictability of residue-residue contacts, probably because in contact prediction the relative ranking of scores (Eqs. 80 or 83 based on couplings is more important than their actual values. ACE with the optimum regularization strength with respect to the reproducibility of fields and couplings showed less accurate contact prediction than plmDCA and mfDCA. For ACE to show comparable performance of contact prediction with plmDCA, regularization strength had to be increased from 
$\gamma=2 / B=10^{-3}$ to $\gamma=1$ for Trypsin inhibitor, making couplings strongly damped and then the generative properties of inferred models lost [8].

Table 2 Free softwares/servers for the direct coupling analysis.

\begin{tabular}{|c|c|c|}
\hline Name & Methods & URL \\
\hline EVcouplings [57] & mfDCA & http://evfold.org \\
\hline EVcouplings, plmc [103 107] & $\mathrm{mf} / \mathrm{plmDCA}$ & https://github.com/debbiemarkslab \\
\hline DCA 71,57 & mfDCA & http://dca.rice.edu/portal/dca/home \\
\hline GaussDCA [7] & GaussDCA & http://areeweb.polito.it/ricerca/cmp/code \\
\hline $\begin{array}{l}\text { FreeContact [47] } \\
\text { plmDCA } \sqrt{23}[22]\end{array}$ & $\begin{array}{l}\text { mfDCA, PSICONV } \\
\text { plmDCA }\end{array}$ & $\begin{array}{l}\text { http://rostlab.org/owiki/index.php/FreeContact } \\
\text { http://plmdca.csc.kth.se/ }\end{array}$ \\
\hline CCMpred [89] & plmDCA & $\begin{array}{l}\text { https:/github.com/pagnani/plmDCA } \\
\text { performance-optimized software } \\
\text { https://github.com/soedinglab/ccmpred }\end{array}$ \\
\hline GREMLIN [6, 48] & GREMLIN & http://gremlin.bakerlab.org/ \\
\hline ACE [15] 16$]$ & ACE & https://github.com/johnbarton/ACE \\
\hline persistent-vi[40] & Persistent VI & https://github.com/debbiemarkslab \\
\hline
\end{tabular}

\subsection{Partial correlation of amino acid cosubstitutions between sites at each branch of a phylogenetic tree}

In the DCA analyses on residue covariations between sites in a multiple sequence alignment (MSA), phylogenetic biases, which are sequence biases due to phylogenetic relations between species, in the MSA must be removed as well as indirect correlations between sites, but instead are reduced by taking weighted averages over homologous sequences in the calculation of single and pairwise frequencies of amino acids; see Eq. 16 .

Needless to say, it is supposed that observed patterns of covariation were caused by molecular coevolution between sites. Whatever caused covariations found in the MSA, it has been confirmed that they can be utilized to predict residue pairs in close proximity in a three dimensional structure. Talavera et al.[99] claimed, however, that covarying substitutions were mostly found on different branches of the phylogenetic tree, indicating that they might or might not be attributable to coevolution.

In order to remove phylogenetic biases and also to respond to such a claim above, it is meaningful to study covarying substitutions between sites in a phylogenetic tree-dependent manner. Such an alternative approach was taken to infer coevolving site pairs from direct correlations between sites in concurrent and compensatory substitutions within the same branches of a phylogenetic tree[66]. In this method, substitution probability and mean changes of physico-chemical properties of side chain accompanied by amino acid substitutions at each site in each branch of the tree are estimated with the likelihood of each substitution to detect concurrent and 
compensatory substitutions. Then, partial correlation coefficients of the vectors of their characteristic changes accompanied by substitutions, substitution probability and mean changes of physico-chemical properties, along branches between sites are calculated to extract direct correlations in coevolutionary substitutions and employed as a score for residue-residue contact. The accuracy of contact prediction by this method was comparable with that by mfDCA [66]. This method, however, has a drawback to be computationally intensive, because an optimum phylogenetic tree must be estimated.

\section{Machine learning methods to augment the contact prediction accuracy based on amino acid coevolution}

Table 3 Machine learning methods that combine predicted direct couplings with other sequence/structure information.

\begin{tabular}{|c|c|c|}
\hline Name & Basic method & Post-processing \\
\hline 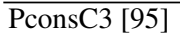 & plmDCA, GaussDCA & 5 layer DNN; http://c3.pcons.net PconsC[94|,C2[96] \\
\hline MetaPSICOV & $\begin{array}{l}\text { PSICOV,mfDCA, } \\
\text { GREMLIN/CCMpred }\end{array}$ & $\begin{array}{l}\text { A two stage neural network predictor; CONSIP2 pipeline } \\
\text { http //bioinf csucl }\end{array}$ \\
\hline RaptorX & CCMpred & $\begin{array}{l}\text { Ultra-deep learning model consisting of } 1 \text { - and } \\
\text { 2-dimensional convolutional residual neural networks } \\
\text { http://raptorx.uchicago.edu/ContactMap/ }\end{array}$ \\
\hline iFold [13] & & Deep neural network (DNN) \\
\hline $\begin{array}{r}\text { EPSILON-CP } \\
\mathrm{mfD}\end{array}$ & $\begin{array}{l}\text { PSICOV, GREMLIN, } \\
\text { CA,CCMpred,GaussDCA }\end{array}$ & $\begin{array}{l}4 \text { hidden layer neural network } \\
\text { with } 400-200-200-50 \text { neurons } 91\end{array}$ \\
\hline
\end{tabular}

All the DCA methods such as mfDCA, plmDCA, GREMLIN, and PSICOV predict significantly nonoverlapping sets of contacts [46, 52, 108]. Then, increasing prediction accuracy by combining their predictions together with other sequence/structure information have been attempted[94, 96, 95, 51, 46, 52, 105, 91]; see Table 3 .

PconsC [94] combines the predictions of PSICOV and plmDCA into a machine learning method, random forests, and employs alignments with HHblits [84] and jackHMMer[42] at four different e-value cut-offs. Five-layer neural network is employed instead of random forests in PconsC2[96], and plmDCA and GaussDCA are employed in PconsC3[95]. A receptive field consisting of $11 \times 11$ predicted contacts around each residue pair is taken into account in each layer except the first one.

MetaPSICOV [46, 52] combines the predictions of PSICOV, mfDCA, and CCMpred/GREMLIN into the first stage of a two-stage neural network predictor together with a well-established "classic" machine learning contact predictor, which utilizes many features such as amino acid profiles, predicted secondary structure and solvent accessibility along with sequence separation predicted, as an additional source 
of information for a little depth of MSAs. The second stage analyses the output of the first stage to eliminate outliers and to fill in the gaps in the contact map. On a set of 40 target domains with a median family size of around 40 effective sequences in CASP11, CONSIP2 server achieved an average top- $L / 5$ long-range contact precision of $27 \%[52]$.

Wang et al.[105] have also shown that a ultra-deep neural network (RaptorX) can significantly improve contact prediction based on amino acid coevolution. They have modeled short-range and long-range correlations in sequential and structural features with respect to complex sequence-structure relationships in proteins by onedimensional and two-dimensional deep neural networks (DNN), respectively. Both the DNNs are convolutional residual neural networks. The 1D DNN performs convolutional transformations, with respect to residue position, of sequential features such as position-dependent scoring matrix, predicted 3-state secondary structure and 3 -state solvent accessibility. The 2D DNN does 2D convolutional transformations of pairwise features such as coevolutional information calculated by CCMpred, mutual information, pairwise contact potentials as well as the output of the 1D DNN converted by a similar operation to outer product. Residual neural networks are employed because they can pass both linear and nonlinear informations from initial input to final output, making their training relatively easy.

\section{Performance of contact prediction}

New statistical methods based on the direct coupling analysis are confirmed in various benchmarking studies[73, 13, 48, 108] to show remarkable accuracy of contact prediction, although deep, stable alignments are required. They can more accurately detect a higher number of contacts between residues, which are very distant along sequence[71]. The top-scoring residue couplings are not only sufficiently accurate but also well-distributed to define the 3D protein fold with remarkable accuracy[57]; this observation was quantified by computing, from sequence alone, all-atom 3D structures of fifteen test proteins from different fold classes, ranging in size from 50 to 260 residues, including a G-protein coupled receptor. The contact prediction performs relatively better on $\beta$ proteins than on $\alpha$ proteins [66]. These initial findings on a limited number of proteins were confirmed as a general trend in a large-scale comparative assessment of contact prediction methods [108, 2].

In CASP12, RaptorX performed the best in terms of F1 score for top $L / 2$ longand medium-range contacts of 38 free-modeling (FM) targets; the total F1 score of RaptorX was better by about 7.6 and $10.0 \%$ than the second and third best servers, iFold_1 and the revised MetaPSICOV, respectively[105, 13]. Tested on 105 CASP11 targets, 76 past CAMEO hard targets, and 398 membrane proteins, the average top $L(L / 10)$ long-range prediction accuracies of RaptorX are $0.47(0.77)$ in comparison with $0.30(0.59)$ for MetaPSICOV and 0.21(0.47) for CCMpred[105, 13]. 


\subsection{MSA dependence of contact prediction accuracy}

In the direct-coupling-based methods, the accuracy of predicted contacts depends on the depth [66, 48, 108] and quality of multiple sequence alignment (MSA) for a target. $5 \times L$ (protein length) aligned sequences may be desirable for accurate contact predictions [48], although attempts to improve prediction methods for fewer aligned sequences have been made 94, 96, 95, 105]. PconsC3 can be used for families with as little as 100 effective sequence members[95]. Also, RaptorX[105] attained top$L / 2$-accuracy $>0.3$ for long-rang contacts even by using MSAs with 20 effective sequence members $\left(M_{\mathrm{eff}} \sim 20\right)$; see Eq. 17 for $M_{\mathrm{eff}}$.

Deepest MSAs including a target sequence were built with various values of Evalue cutoff[94] and coverage parameters [46, 52] in sequence search and alignment programs based on the hidden Markov models such as HHblits and jackHMMer. Although prediction performance tends to increase in general as alignment depth is deeper[66], it was reported[52] that in the case of transmembrane domains, building too deep alignments could result in unrelated sequences or drifted domains being included. To increase alignment quality, E-value and coverage parameters may be carefully tuned for each alignment[52]. In the case of alignments that might contain regions of partial matches, a too stringent sequence coverage requirement could result in missing related sequences. On the other hand, a too permissive sequence coverage requirement could pick up unrelated sequences, permitting many partial matches. A trade-off is required between the effective number of sequences and sequence coverage, and an appropriate E-value must be chosen not to much decrease both alignment depth and sequence coverage[37].

\section{Contact-guided de novo protein structure prediction}

It is a primary obstacle to de novo structure prediction that current methods and computers cannot make it feasible to adequately sample the vast conformational space a protein might take in the precess of folding into the native structure [49]. Thus, it is critical whether residue-residue proximities inferred with direct coupling analysis can provide sufficient information to reduce a huge search space for a protein fold, without any known 3D structural information of the protein.

Algorithms are needed to fold proteins into native folds based on contact information; see Table 4. Distance geometry generation[36, 9] of 3D structures, which may be followed by energy minimization and molecular dynamics, will be just the primary one. In EVfold[57], contacts inferred by direct coupling analysis and predicted secondary structure information are translated into a set of distance constraints for the use of a distance geometry algorithm in the Crystallography and NMR System (CNS)[10]. It was confirmed that the evolutionary inferred contacts can sufficiently reduce a search space in the structure predictions of 15 test proteins from different fold classes[57], and of 11 unknown and 23 known transmembrane protein structures[37]. Because distance constraints from predicted contacts may 
Table 4 Contact-guided de novo protein structure prediction methods and servers.

\begin{tabular}{|c|c|c|}
\hline Name & Contact prediction & \\
\hline $\begin{array}{l}\text { EVfold [57 [58] } \\
\text { /EVfold_men }\end{array}$ & $\begin{array}{l}\text { mfDCA / plmDCA } \\
\text { abrane [37] }\end{array}$ & $\begin{array}{l}\text { Using distance geometry algorithm[36] and } \\
\text { simulated annealing of CNS[10]; http://evfold.org/ }\end{array}$ \\
\hline DCA-fold 97 & mfDCA & $\begin{array}{l}\text { Simulated annealing using a coarse-grained } \\
\text { molecular dynamics for a } \mathrm{C}_{\alpha} \text { model }\end{array}$ \\
\hline $\begin{array}{l}\text { FRAGFOLD } \\
\text { /FILM3 }\end{array}$ & MetaPSICOV & $\begin{array}{l}\text { Combining fragment-based folding algorithm[44] } \\
\text { with PSICOV[51] and with MetaPSICOV[46]. } \\
\text { FILM3[75] is employed instead of FRAGFOLD [43] } \\
\text { for transmembrane proteins. }\end{array}$ \\
\hline CONFOLD [1] & $\begin{array}{l}\text { EVFOLD / FRAGFOLD } \\
\text { (PSIPRED for 2nd structures }\end{array}$ & $\begin{array}{l}\text { Two-stage contact-guided de novo protein folding, } \\
\text { using distance geometry simulated annealing } \\
\text { protocol in a revised CNS v1.3. } \\
\text { http://protein.rnet.missouri.edu/confold/| }\end{array}$ \\
\hline Rosetta [50][77] & GREMLIN & Fragment assembly \\
\hline
\end{tabular}

be partial in a protein sequence, they should be embedded into ab initio structure prediction methods.

Sułkowska et al. also showed that a simple hybrid method, called DCA-fold, integrating mfDCA-predicted contacts with an accurate knowledge of secondary structure is sufficient to fold proteins in the range of 1-3 $\AA$ resolution[97]. In this study, simulated annealing using a coarse-grained molecular dynamics model was employed for a $\mathrm{C}_{\alpha}$ chain model, in which $\mathrm{C}_{\alpha} \mathrm{s}$ interact with each other with a contact potential approximated by a Gaussian function and a torsional potential depending on $\mathrm{C}_{\alpha}$ dihedral angles at each position.

Adhikari et al.[1] studied a way to effectively encode secondary structure information into distance and dihedral angle constrains that complement long-range contact constraints, and revised the CNS v1.3 to effectively use secondary structure constraints together with predicted long-range constraints; CONFOLD[1] consists of two stages. In the first stage secondary structure information is converted into distance, dihedral angle, and hydrogen bond constraints, and then best models are selected by executing the distance geometry simulated annealing. In the second stage self-conflicting contacts in the best structure predicted in the first stage are removed, constrains based on the secondary structures are refined, and again the distance geometry simulated annealing is executed.

Baker group[77] embedded contact constraints predicted by GREMLIN[48] as sigmoidal constraints to overcome noise in the Rosetta[50] conformational sampling and refinement. They found that model accuracy will be generally improved, if more than $3 \mathrm{~L}$ (protein length) sequences are available, and that large topologically complex proteins can be modeled with close to atomic-level accuracy without knowledge of homologous structures, if there are enough homologous sequences available.

On the other hand, a fragment-based folding algorithm FRAGFOLD was combined with PSICOV [51] and with MetaPSICOV [46, 52]; In this approach, predicted 
contacts are converted into additional energy terms for FRAGFOLD in addition to the pairwise potentials of mean force and solvation [46, 52]. FILM3[75], with constraints based on predicted contacts and ones approximating Z-coordinate values within the lipid membrane, is employed instead of FRAGFOLD for transmembrane proteins.

RaptorX [105] employed the CNS suite[10] to generate 3D models from predicted contacts and secondary structure converted to distance, angle and h-bond restraints, and could yield TMscore $>0.6$ for 203 of 579 test proteins, while using MetaPSICOV and CCMpred could do so for 79 and 62, respectively.

\subsection{How many predicted contacts should be used to build 3D models?}

The number of feasible contacts surrounding a residue in a protein is about 6.3 [70], which corresponds to the maximum number of contacts per a protein, $6.3 \mathrm{~L} / 2$, where $L$ denotes protein length. However, more than $50 \%$ of known 3D structures in the PDB have less than $2 L$ contacts, and in the test on 15 proteins in EVfold benchmark set, less than $1.6 \mathrm{~L}$ predicted contacts yielded best results[1]. In the original EVfold, the optimal number of evolutionary constraints was in the order of $0.5 \mathrm{~L}$ to $0.7 L[37]$. Because prediction accuracy tends to decrease as the rank of contact score increases, and different proteins need different numbers of predicted contacts to be folded well, protein folds were generated with a wide range of the number of predicted contacts, and then best folds were selected; from 30 to $L$ in EVfold[37], and from $0.4 L$ to $2.2 L$ in CONFOLD[1]. In RaptorX, the top $2 L$ predicted contacts irrespective of site separation were converted to distance restraints[105]. On the other hand, Jones group reported[51] that artificially truncating the list of predicted contacts was likely to remove useful information to fold a protein with FRAGFOLD and PSICOV, in which the weight of a given predicted contact is determined by its positive predictive value; see section 9.2.9

\section{Evolutionary direct couplings between residues not contacting in a protein 3D structure}

Needless to say, evolutionary constraints do not only originate in intra-molecular contacts but also result from inter-molecular contacts/interactions. Even in the case of intra-molecular contacts, if there are structural variations including ones due to conformational changes in a protein family, evolutionary constraints will reflect the alternative conformations [71, 37, 4]. Also, intra-molecular residue couplings may contain useful information of ligand-mediated residue couplings [71, 77]. On the other hand, inter-molecular contacts may allow us to predict protein complexes, and are useful to build protein-protein interaction networks at a residue level. 


\subsection{Structural variation including conformational changes}

MSA contains information on all members of the protein family, and direct couplings between residues estimated from the MSA reflect the structures of all members. It was shown[4] that $74 \%$ of top $L / 2$ direct couplings residue pairs that are more than $5 \AA$ apart in the target structures of 3883 proteins are less than $5 \AA$ apart in at least one homolog structure.

Conformational change is an interesting case of structural variation. Many proteins adopt different conformations as part of their functions[102], indicating that protein flexibility is as important as structure on biological function. Protein flexibility around the energy minimum can be studied by sampling around the native structure in normal mode/principal component analysis, coarse-grained elastic network model, and short-timescale MD simulations. However, distant conformers that require large conformational transitions are difficult to predict. If conformational changes are essential on protein functions, evolutionary constraints will reflect the multiple conformations. Toth-Petroczy et al.[103] showed that coevolutionary information may reveal alternative structural states of disorderd regions.

Morcos et al.[71] found that some of top predicted contacts in the responseregulator DNA-binding domain family (GerE, PF00196) conflict with the structure (PDB ID 3C3W) of the full-length response-regulator DosR of M. tuberculosis, but are compatible with the structure (PDB ID 1JE8) of DNA-binding domain of $E$. coli NarL.

Sutto et al.[98] combined coevolutionary data and molecular dynamics simulations to study protein conformational heterogeneity; the Boltzmann-learning algorithm with $\ell_{2}$ regularization terms was employed to extract direct couplings between sites in homologous protein sequences, and a set of conformations consistent with the observed residue couplings were generated by exhaustive sampling simulations based on a coarse-grained protein model. Although the most representative structure was consistent with the experimental fold, the various regions of the sequence showed different stability, indicating conformational changes[98].

Sfriso et al.[90] made an automated pipeline based on discrete molecular dynamics guided by predicted contacts for the systematic identification of functional conformations in proteins, and identified alternative conformers in 70 of 92 proteins in a validation set of proteins in PDB; various conformational transitions are relevant to those conformers, such as open-closed, rotation, rotation-closed, concerted, and miscellanea of complex motions.

\subsection{Homo-oligomer contacts}

Intra-molecular contacts that conflict with the native fold may indicate homooligomer contacts [4]. Such a case was confirmed for homo-oligomer contacts in the ATPase domain of nitrogen regulatory protein C-like sigma-54 dependent transcriptional activators [71] and between transmembrane helices [37]. It was pointed out [37] 
that the identification of evolutionary couplings due to homo-oligomerization is not only meaningful in itself but also useful because their removal improves the accuracy of the structure prediction for the monomer.

\subsection{Residue couplings mediated by binding to a third agent}

Direct couplings between residues found by the DCA analysis can be mediated[71] by their interactions with a third agent, i.e., ligands, substrates, RNA, DNA, and other metabolites. This indicates that binding sites with such a agent may be found as residue sites directly coupled but not in contact.

If interactions with a third agent requires too specific residue type at a certain site, then the residue type will be well conserved at the binding sites. This often occurs, and has been utilized to identify binding sites. However, the interactions for binding are less specific but certainly restricted, direct couplings between residues around the binding sites may occurs.

Hopf et al.[37] devised a total evolutionary coupling score, which is defined as EC values summed over all high-ranking pairs involving a given residue and normalized by their average over all high-ranking pairs, and showed that residues with high total coupling scores line substrate-binding sites and affect signaling or transport in transmembrane proteins, Adrb2 and Opsd.

\section{Heterogeneous protein-protein contacts}

An application of the direct coupling analysis to predict the structures of protein complexes is straightforward. In place of a MSA of a single protein family, a single MSA that is built by concatenating the multiple MSAs of multiple protein families every species can be employed to extract direct couplings between sites of different proteins by removing indirect intra- and inter-protein couplings [78, 93, 106, 37].

A critical requirement for sequences to be concatenated is, however, that respective sets of the protein sequences must have the same evolutionary history to coevolve. In other words, phylogenetic trees built from the respective sets of sequences employed for the protein families must have at least the same topology. One way to build a set of cognate pairs of protein sequences is to employ orthologous sequences for each protein family, the phylogenetic tree of which coincides with that of species. Thus, a genome-wide analysis of finding protein-protein interactions based on protein sequences is not so simple.

Weigt et al.[106] successfully applied the direct coupling analysis to the bacterial two-component signal transduction system consisting of sensor kinase (SK) and response regulator (RR), which are believed[93] to interact specifically with each other in most cases and often revealed by adjacency in chromosomal location. This analysis is based on the fact that in prokaryotes cognate pairs are often encoded 
in the same operon. Genome-sequencing projects have revealed that most organisms contain large expansions of a relatively small number of signaling families[93]. However, it is not as simple as in prokaryotes to build a set of cognate pairs of those protein sequences in eukaryotes.

Hopf et al.[39] developed a contact score, EVcomplex, for every inter-protein residue pair based on the overall inter-protein EC score distributions, evaluated its performance in blinded tests on 76 complexes of known 3D structure, predicted protein-protein contacts in 32 complexes of unknown structure, and then demonstrated how evolutionary direct couplings can be used to distinguish between interacting and non-interacting protein pairs in a large complex; see section 9.2 .9 for EC score. In their analysis, protein sequence pairs that are encoded close on E. coli genome were employed to reduce incorrect protein pairings.

\section{Discussion}

Determination of protein structure is essential to understand protein function. However, despite significant effort to explore unknown folds in the protein structural space, protein structures determined by experiment are far less than known protein families. Only about 41-42\% of the Pfam families[27] (Pfam-A release 31.0, 16712 families) include at least one member whose structure is known. The number and also the size of protein families will further grow as genome/metagenome sequencing projects proceed with next-generation sequencing technologies. Thus, accurate de novo prediction of three-dimensional structure is desirable to catch up with the high growing speed of protein families with unknown folds. Coevolutionary information can be used to predict not only proteins but also RNAs[107] and those complexes, together with experimental informations such as X-ray, NMR, SAS, FRET, crosslinking, Cryo-EM, and others.

Here, statistical methods for disentangling direct from indirect couplings between sites with respect to evolutionary variations/substitutions of amino acids in homologous proteins have been briefly reviewed. Dramatic improvements on contact prediction and successful 3D de novo predictions based on predicted contacts are described in details in the recent reports of CASP-11[73] and CASP-12 meetings [13]. Machine learning methods, particularly deep neural network (DNN) such as MetaPSICOV, iFold, and RaptorX, have shown to significantly augment contact prediction accuracy based on coevolutionary information. However, the present state-of-the-art DNN methods are, at least at the very moment, not powerful enough to extract coevolutionary information directly from homologous sequences. It was reported that without coevolutionary strength produced by CCMpred the top $L / 10$ long-range prediction accuracy of RaptorX might drop by 0.15 for soluble proteins and more for membrane proteins [105], indicating that the direct coupling analysis is still essential for contact prediction.

The primary requirement for the direct coupling analysis is a high quality deep alignment. However, genome/metagenome sequencing projects provide more ge- 
netic variations from which more accurate and more comprehensive information on evolutionary constraints can be extracted. One of problems is that species being sequenced may be strongly biased to prokaryotes, making it hard to analyze eukaryotic proteins based on coevolutionary substitutions. Experiments of vitro evolution may be useful to provide sequence variations for eukaryotic proteins [77].

For a large-scale of protein structure prediction, computationally intensive methods such as the ACE and Boltzmann machine (MCMC and mpDCA) can hardly be employed. The Gaussian approximation with a normal-inverse-Wishart prior, the Gaussian approximations with other priors (PSICOV) and mean field approximation (mfDCA) are fast enough but their performance of contact prediction tends to be compared unfavorably with the pseudo-likelihood approximation (plmDCA), indicating that they may be inappropriate for proteins with sparse couplings.

The accurate estimates of fields and couplings are very informative in evaluating the effects $\left(\Delta H_{\text {Potts }}\right)$ of mutations [38], identifying protein family members and also studying folding mechanisms[72, 41] and protein evolution[68]. It should be also examined whether the distribution of dimensionless energies $\left(H_{\text {Potts }}\right)$ over homologous proteins can be well reproduced. Accuracy of estimates of fields and couplings and the distribution of dimensionless energies depends on regularization parameters or the ratio of pseudocount [8, 68], and therefore they should be optimized. It was also pointed out that group $L_{1}$ regularization performs better than $L_{2}$ for the maximum pseudolikelihood method[40]. The ACE algorithm, which can be applied only for systems of sparse couplings, may be more favorable with respect to computational load for the estimation of fields and couplings than Boltzmann learning with Monte Carlo simulation or with message passing. However, both the methods are computationally intensive. Recently, another approach consisting of two methods named persistent-vi and Fadeout, in which the posterior probability density with horseshoe prior is approximately estimated by using variational inference and noncentered parameterization for such a sparsity-inducing prior, has shown to perform better with twofold cpu time than the maximum pseudolikelihood method with $L_{2}$ and group $L_{1}$ regularizations [40].

The remarkable advances of sequencing technologies and also statistical methods are likely to bring many targets within range of the present approach in the near future, and have a potential to transform the field[73].

\section{Appendix}

\subsection{Direct Coupling Analysis for amino acid covariations between sites}

\subsubsection{A reweighted-sampling scheme to reduce phylogenetic biases in a MSA}

In statistical approaches described in this section, sequences in a MSA are assumed to be independently and identically distributed samples in sequence space. However, homologous sequences in a 
MSA are actually biased due to phylogenetic relations between species. Such phylogenetic biases are reduced here by taking weighted averages over the sequences in calculation of single and pairwise amino acid frequencies. The amino acid frequencies $f_{i}\left(a_{k}\right)$ at site $i$ and pairwise frequencies $f_{i j}\left(a_{k}, a_{l}\right)$ between sites $i$ and $j$ are calculated as follows.

$$
\begin{aligned}
f_{i}\left(a_{k}\right) & =\frac{1}{M_{\mathrm{eff}}} \sum_{\tau} w_{\sigma^{\tau}} \delta_{\sigma_{i}^{\tau} a_{k}} \quad, \quad f_{i j}\left(a_{k}, a_{l}\right)=\frac{1}{M_{\mathrm{eff}}} \sum_{\tau} w_{\sigma^{\tau}} \delta_{\sigma_{i}^{\tau} a_{k}} \delta_{\sigma_{j}^{\tau} a_{l}} \\
w_{\sigma} & =1 /\left[\sum_{\tau} \theta\left(\sum_{i=1}^{L} \delta_{\sigma_{i} \sigma_{i}^{\tau}}-s L\right)\right] \quad, \quad B=M_{\mathrm{eff}} \equiv \sum_{\tau} w_{\sigma^{\tau}}
\end{aligned}
$$

where $\theta$ is a Heaviside step function, and $s$ is a similarity threshold for sequence identity to regard two sequences virtually identical; $s=0.8[71]$ and $s=0.7[57][105]$ were employed. Thus, $B$ in Eq. 7 is taken here to be equal to $M_{\text {eff }}$ that is the effective number of sequences. In GaussDCA[7], the threshold $1-s$ is defined as being inversely proportional to the average sequence identity over all pairs of sequences; $s=1-0.1216$ / average-sequence-identity.

Except for models in which priors or regularization terms are not taken into account, these corrected frequencies are employed as single-site and two-site marginal probabilities;

$$
P_{i}\left(a_{k}\right)=f_{i}\left(a_{k}\right) \quad P_{i j}\left(a_{k}, a_{l}\right)=f_{i j}\left(a_{k}, a_{l}\right)
$$

In the models with $R=0$, pseudocount based on Bayesian statistics, which is a correction scheme for small sample size, is employed to estimate $P_{i}\left(a_{k}\right)$ and $P_{i j}\left(a_{k}, a_{l}\right)$; see Eqs. 43 and 44

\subsection{Inverse Potts model}

\subsubsection{A gauge employed for $h_{i}\left(a_{k}\right)$ and $J_{i j}\left(a_{k}, a_{l}\right)$}

Unless specified, a following gauge is employed; we call it $q$-gauge, here.

$$
h_{i}\left(a_{q}\right)=J_{i j}\left(a_{k}, a_{q}\right)=J_{i j}\left(a_{q}, a_{l}\right)=0
$$

In this gauge, the amino acid $a_{q}$ is the reference state for fields and couplings, and $P_{i}\left(a_{q}\right)$, $P_{i j}\left(a_{k}, a_{q}\right)=P_{j i}\left(a_{q}, a_{k}\right)$, and $P_{i j}\left(a_{q}, a_{q}\right)$ are regarded as dependent variables. Common choices for the reference state $a_{q}$ are the most common (consensus) state at each site. Any gauge can be transformed to another by the following transformation.

$$
\begin{aligned}
J_{i j}^{\mathrm{I}}\left(a_{k}, a_{l}\right) & \equiv J_{i j}\left(a_{k}, a_{l}\right)-J_{i j}\left(\cdot, a_{l}\right)-J_{i j}\left(a_{k}, \cdot\right)+J_{i j}(\cdot, \cdot) \\
h_{i}^{\mathrm{I}}\left(a_{k}\right) & \equiv h_{i}\left(a_{k}\right)-h_{i}(\cdot)+\sum_{j \neq i}\left(J_{i j}\left(a_{k}, \cdot\right)-J_{i j}(\cdot, \cdot)\right)
\end{aligned}
$$

where "." denotes the reference state, which may be $a_{q}$ for each site (q-gauge) or the average over all states (Ising gauge).

\subsubsection{Boltzmann machine}

Fields $h_{i}\left(a_{k}\right)$ and couplings $J_{i j}\left(a_{k}, a_{l}\right)$ are estimated by iterating the following 2-step procedures. 
1. For a given set of $h_{i}$ and $J_{i j}\left(a_{k}, a_{l}\right)$, marginal probabilities, $P^{\mathrm{MC}}\left(\sigma_{i}=a_{k}\right)$ and $P^{\mathrm{MC}}\left(\sigma_{i}=\right.$ $a_{k}, \sigma_{j}=a_{l}$ ), are estimated by a Markov chain Monte Carlo method (the Metropolis-Hastings algorithm[60]) or by any other method (for example, the message passing algorithm[106]).

2. Then, $h_{i}$ and $J_{i j}\left(a_{k}, a_{l}\right)$ are updated according to the gradient of negative log-posterior-probability per instance, $\partial S_{0} / \partial h_{i}\left(a_{k}\right)$ or $\partial S_{0} / \partial J_{i j}\left(a_{k}, a_{l}\right)$, multiplied by a parameter-specific weight factor [8], $w_{i}\left(a_{k}\right)$ or $w_{i j}\left(a_{k}, a_{l}\right)$; see Eqs. 8 and 12

$$
\begin{aligned}
\Delta h_{i}\left(a_{k}\right) & =-\left(P^{\mathrm{MC}}\left(\sigma_{i}=a_{k}\right)+\frac{\partial R}{\partial h_{i}\left(a_{k}\right)}-P_{i}\left(a_{k}\right)\right) \cdot w_{i}\left(a_{k}\right) \\
\Delta J_{i j}\left(a_{k}, a_{l}\right) & =-\left(P^{\mathrm{MC}}\left(\sigma_{i}=a_{k}, \sigma_{j}=a_{l}\right)+\frac{\partial R}{\partial J_{i j}\left(a_{k}, a_{l}\right)}-P_{i j}\left(a_{k}, a_{l}\right)\right) \cdot w_{i j}\left(a_{k}, a_{l}\right)
\end{aligned}
$$

where weights are also updated as $w_{i}\left(a_{k}\right) \leftarrow f\left(w_{i}\left(a_{k}\right)\right)$ and $w_{i j}\left(a_{k}, a_{l}\right) \leftarrow f\left(w_{i j}\left(a_{k}, a_{l}\right)\right)$ according to the RPROP[85] algorithm; the function $f(w)$ is defined as

$$
f(w) \equiv \begin{cases}\max \left(w \cdot s_{-}, w_{\min }\right) & \text { if the gradient changes its sign, } \\ \min \left(w \cdot s_{+}, w_{\max }\right) & \text { otherwise }\end{cases}
$$

The $w_{\text {min }}=10^{-3}, w_{\max }=10, s_{-}=0.5$, and $s_{+}=1.9<1 / s_{-}$were employed[8]. After updated, $h_{i}\left(a_{k}\right)$ and $J_{i j}\left(a_{k}, a_{l}\right)$ may be modified to satisfy a given gauge.

The Boltzmann machine has a merit that model correlations are calculated.

\subsubsection{Message passing algorithm to estimate marginal probabilities}

To estimate marginal probabilities for given fields and couplings, the standard belief propagation algorithm for single-site marginals and the generalized belief propagation (susceptibility propagation 61]) algorithm for two-site marginals were employed in mpDCA 106]; these algorithms are exact for trees but approximate for general graphs that is the present case.

Belief propagation algorithm to estimate single-site marginals, $\left\{P^{\mathrm{MP}}\left(\sigma_{i}=a_{k}\right)\right\}$

In this algorithm for single-site marginals, messages (beliefs) $P_{i \rightarrow m}^{\mathrm{MP}}\left(a_{k}\right)$, which may be understood as the marginal distribution at site $i$ in the system where site $m$ is removed, are self-consistently passed between sites. The self-consistent messages can be obtained by iteratively solving the following equations with arbitrary initial messages for the Hamiltonian defined in Eq. 5 i.e., given $h^{\mathrm{MP}}$ and $J^{\mathrm{MP}}$.

$$
P_{i \rightarrow m}^{\mathrm{MP}}\left(a_{k}\right) \propto \exp \left(h_{i}^{\mathrm{MP}}\left(a_{k}\right)\right) \prod_{j \neq i, m}\left[\sum_{l} \exp \left(J_{j i}^{\mathrm{MP}}\left(a_{l}, a_{k}\right)\right) P_{j \rightarrow i}^{\mathrm{MP}}\left(a_{l}\right)\right]
$$

with $\sum_{k} P_{i \rightarrow m}^{\mathrm{MP}}\left(a_{k}\right)=1$. Random sequential updates seem to be most efficient for solving[106].

Having calculated all messages, true single-site marginals can be estimated by

$$
P^{\mathrm{MP}}\left(\sigma_{i}=a_{k}\right) \propto \exp \left(h_{i}^{\mathrm{MP}}\left(a_{k}\right)\right) \prod_{j \neq i}\left[\sum_{l} \exp \left(J_{j i}^{\mathrm{MP}}\left(a_{l}, a_{k}\right)\right) P_{j \rightarrow i}^{\mathrm{MP}}\left(a_{l}\right)\right]
$$

After convergence the fields can be determined from Eq. 26] as

$$
\left.\exp \left(h_{i}^{\mathrm{MP}}\left(a_{k}\right)\right) \propto \frac{P_{i}\left(a_{k}\right)}{\prod_{j \neq i}\left[\sum_{l} \exp \left(J_{j i}^{\mathrm{MP}}\left(a_{l}, a_{k}\right)\right) P_{j \rightarrow i}^{\mathrm{MP}}\left(a_{l}\right)\right.}\right]
$$


The fields $h_{i}^{\mathrm{MP}}\left(a_{k}\right)$ must be fixed by a given gauge condition. Eq. 27 guarantees that the singlesite marginals agree to given amino acid frequencies $\left(P^{\mathrm{MP}}\left(\sigma_{i}=a_{k}\right)=P_{i}\left(a_{k}\right)\right)$ and therefore the gradient descent update for fields is not needed[106]. This characteristic is useful in the inference (gradient descent updates) of couplings for Potts models with $q>3$ that tend to show first-order phase transitions, and in which small changes in fields and couplings may lead to large changes in marginals 106.

Susceptibility propagation algorithm to estimate two-site marginals, $\left\{P^{\mathrm{MP}}\left(\sigma_{i}=a_{k}, \sigma_{j}=a_{l}\right)\right\}$

After all single-site marginals are calculated for a given Hamiltonian, the covariance matrix, which includes two-site marginals, can be estimated after iterations to obtain self-consistent messages in the equation of susceptibility propagation that is derived from Eq. 25 as follows.

$$
\begin{gathered}
M_{i \rightarrow m ; j}^{\mathrm{MP}}\left(a_{k}, a_{l}\right) \equiv \frac{\partial P_{i \rightarrow m}^{\mathrm{MP}}\left(a_{k}\right)}{\left.\partial h_{j}^{\mathrm{MP}}\left(a_{l}\right)\right)}=P_{i \rightarrow m}^{\mathrm{MP}}\left(a_{k}\right)\left[\frac{\partial \log f_{i \rightarrow m}\left(a_{k}\right)}{\left.\partial h_{j}^{\mathrm{MP}}\left(a_{l}\right)\right)}-\sum_{k} P_{i \rightarrow m}^{\mathrm{MP}}\left(a_{k}\right) \frac{\partial \log f_{i \rightarrow m}\left(a_{k}\right)}{\left.\partial h_{j}^{\mathrm{MP}}\left(a_{l}\right)\right)}\right] \\
\frac{\partial \log f_{i \rightarrow m}\left(a_{k}\right)}{\left.\partial h_{j}^{\mathrm{MP}}\left(a_{l}\right)\right)}=\left[\delta_{i j} \delta_{k l}+\sum_{n \neq i, m} \frac{\sum_{v} \exp \left(J_{n i}^{\mathrm{MP}}\left(a_{v}, a_{k}\right) M_{n \rightarrow i ; j}^{\mathrm{MP}}\left(a_{v}, a_{l}\right)\right.}{\sum_{v} \exp \left(J_{n i}^{\mathrm{MP}}\left(a_{v}, a_{k}\right) P_{n \rightarrow i}^{\mathrm{MP}}\left(a_{v}\right)\right.}\right]
\end{gathered}
$$

where the second term of Eq. 28 is a term that results from the partial derivative of the normalization of $P_{i \rightarrow m}^{\mathrm{MP}}\left(a_{k}\right)$ in Eq. 25 Then, similarly the covariance matrix can be estimated as follows

$$
\begin{aligned}
\frac{\partial P^{\mathrm{MP}}\left(\sigma_{i}=a_{k}\right)}{\partial h_{j}^{\mathrm{MP}}\left(a_{l}\right)} & =P^{\mathrm{MP}}\left(\sigma_{i}=a_{k}, \sigma_{j}=a_{l}\right)-P_{i}\left(a_{k}\right) P_{j}\left(a_{l}\right) \\
& =P_{i}\left(a_{k}\right)\left[\frac{\partial \log f_{i}\left(a_{k}\right)}{\left.\partial h_{j}^{\mathrm{MP}}\left(a_{l}\right)\right)}-\sum_{k} P_{i}\left(a_{k}\right) \frac{\partial \log f_{i}\left(a_{k}\right)}{\left.\partial h_{j}^{\mathrm{MP}}\left(a_{l}\right)\right)}\right] \\
\frac{\partial \log f_{i}\left(a_{k}\right)}{\left.\partial h_{j}^{\mathrm{MP}}\left(a_{l}\right)\right)} & =\left[\delta_{i j} \delta_{k l}+\sum_{n \neq i} \frac{\sum_{v} \exp \left(J_{n i}^{\mathrm{MP}}\left(a_{v}, a_{k}\right) M_{n \rightarrow i ; j}^{\mathrm{MP}}\left(a_{v}, a_{l}\right)\right.}{\sum_{v} \exp \left(J_{n i}^{\mathrm{MP}}\left(a_{v}, a_{k}\right) P_{n \rightarrow i}^{\mathrm{MP}}\left(a_{v}\right)\right.}\right]
\end{aligned}
$$

In the equation above, $P^{\mathrm{MP}}\left(\sigma_{i}=a_{k}\right)=P_{i}\left(a_{k}\right)$ is employed, because it is guaranteed by Eq. 27

It requires very intensive calculations to obtain self-consistent $M_{i \rightarrow m ; j}^{\mathrm{MP}}\left(a_{k}, a_{l}\right)$, because the number of the messages is $O\left(L^{3} q^{2}\right)$, and an efficient implementation requires $O\left(L^{4} q^{2}\right)$ steps of calculations for a given set of fields $h_{i}^{\mathrm{MP}}\left(a_{k}\right)$ and couplings $J_{i j}^{\mathrm{MP}}\left(a_{k}, a_{l}\right)$ [106.

\subsubsection{Mean field approximation for the inverse Potts model}

In this approximation, no prior knowledge $\left(P_{0}=\right.$ constant $)$ or the limit of a infinite number of instances $(B \rightarrow \infty)$ is assumed; the regularization term $R=0$ in Eq. $8 B \rightarrow \infty$ is reasonable for most problems in physics. For the present case, however, pseudocount must be employed because of a small number of instances. 
$h_{i}^{\mathrm{MF}}\left(a_{k}\right)$ in the mean field approximation

The partition function is expanded in terms of coupling $J$ by representing it as a function of $\alpha$ as follows 79 .

$$
-F(\alpha) \equiv \log Z(\alpha)=\log Z^{\mathrm{MF}}(\alpha)+O\left(\alpha^{2}\right)
$$

where

$$
Z(\alpha) \equiv \sum_{\sigma} \exp \left[+\sum_{i} h_{i}\left(\sigma_{i}\right)+\frac{\alpha}{2} \sum_{i} \sum_{j \neq i} J_{i j}\left(\sigma_{i}, \sigma_{j}\right)\right]
$$

Then, ignoring the terms of $\alpha^{n}$ with $n>1$, the partition function in the mean field approximation, which would be adequate to the case of weak couplings, is obtained.

$$
\begin{aligned}
& -F^{\mathrm{MF}}(1) \equiv \log Z^{\mathrm{MF}}(1) \\
& =-\sum_{i} \sum_{k} P_{i}\left(a_{k}\right) \log P_{i}\left(a_{k}\right)+\sum_{i} \sum_{k}\left[h_{i}\left(a_{k}\right) P_{i}\left(a_{k}\right)+\frac{1}{2} \sum_{j \neq i} \sum_{l} J_{i j}\left(a_{k}, a_{l}\right) P_{i}\left(a_{k}\right) P_{j}\left(a_{l}\right)\right]
\end{aligned}
$$

$P_{i}\left(a_{k}\right)$ minimizing $F^{\mathrm{MF}}\left(P_{i}\left(a_{k}\right)\right)$ satisfies

$$
P_{i}\left(a_{k}\right)=\frac{\exp \left(h_{i}^{\mathrm{MF}}\left(a_{k}\right)+\sum_{j \neq i} \sum_{l \neq q} J_{i j}^{\mathrm{MF}}\left(a_{k}, a_{l}\right) P_{j}\left(a_{l}\right)\right)}{1+\sum_{k \neq q} \exp \left(h_{i}^{\mathrm{MF}}\left(a_{k}\right)+\sum_{j \neq i} \sum_{l \neq q} J_{i j}^{\mathrm{MF}}\left(a_{k}, a_{l}\right) P_{j}\left(a_{l}\right)\right)}
$$

This famous mean field equation can be utilized to estimate fields $h_{i}^{\mathrm{MF}}\left(a_{k}\right)$ from marginal probabilities $\left(P_{i}\left(a_{k}\right)\right)$ and couplings $J_{i j}^{\mathrm{MF}}\left(a_{k}, a_{l}\right)$, which are derived in the next paragraph.

$J_{i j}^{\mathrm{MF}}\left(a_{k}, a_{l}\right)$ in the mean field approximation

The free energy $F$ for the Potts model satisfies

$$
d F(h, J)=-\sum_{i} \sum_{k \neq q} P_{i}\left(a_{k}\right) d h_{i}\left(a_{k}\right)-\sum_{i} \sum_{k \neq q} \sum_{j>i} \sum_{l \neq q} P_{i j}\left(a_{k}, a_{l}\right) d J_{i j}\left(a_{k}, a_{l}\right)
$$

We transform variables from $h_{i}$ to $P_{i}$ by a Legendre transformation as follows [31].

$$
-G\left(h,\left\{P_{i j}\right\} \mid\left\{P_{i}\right\}, J\right)=\log Z\left(\left\{P_{i}\right\}, J\right)-\sum_{i} \sum_{k \neq q} h_{i}\left(a_{k}\right) P_{i}\left(a_{k}\right)
$$

The given covariance matrix $C$ can be represented as follows.

$$
\begin{aligned}
C_{i(q-1)+k, n(q-1)+m} & \equiv C_{i n}\left(a_{k}, a_{m}\right) \equiv P_{i n}\left(a_{k}, a_{m}\right)-P_{i}\left(a_{k}\right) P_{n}\left(a_{m}\right) \\
& =\frac{\partial^{2}-F(h, J)}{\partial h_{n}\left(a_{m}\right) \partial h_{i}\left(a_{k}\right)}=\left(\frac{\partial P_{i}\left(a_{k}\right)}{\partial h_{n}\left(a_{m}\right)}\right)_{\left\{h_{i}\right\},\left\{J_{i j}\right\}} \\
\left(C^{-1}\right)_{i(q-1)+k, n(q-1)+m} & \equiv\left(C^{-1}\right)_{i n}\left(a_{k}, a_{m}\right)=\left(\frac{\partial^{2} G\left(\left\{P_{i}\right\}, J\right)}{\partial P_{i}\left(a_{k}\right) \partial P_{n}\left(a_{m}\right)}\right)=\left(\frac{\partial h_{i}\left(a_{k}\right)}{\partial P_{n}\left(a_{m}\right)}\right)_{\left\{P_{i}\right\},\left\{J_{i j}\right\}}
\end{aligned}
$$

From $h_{i}\left(a_{k}\right) \simeq h_{i}^{\mathrm{MF}}\left(a_{k}\right)$, Eqs. 36 and 41 the following equation for couplings $J_{i j}^{\mathrm{MF}}\left(a_{k}, a_{l}\right)$ is derived.

$$
-\left(C^{-1}\right)_{i n}\left(a_{k}, a_{m}\right) \simeq J_{i n}^{\mathrm{MF}}\left(a_{k}, a_{m}\right)\left(1-\delta_{i, n}\right)-\delta_{i n}\left(\frac{\delta_{k m}}{P_{n}\left(a_{m}\right)}+\frac{1}{P_{n}\left(a_{q}\right)}\right)
$$




\section{Pseudocount to make the covariance matrix invertible}

Couplings $J^{\mathrm{MF}}$ in the mean field approximation is equal to $-C^{-1}$ except diagonal $(q-1) \times(q-1)$ matrices; see Eq. 42 In the case of protein families, the depth of MSA is very limited, $B<(q-1) L$, and therefore the observed covariance matrix $C$ is singular and must be modified to be regular. Here, pseudocount based on Bayesian statistics is employed[106].

$$
\begin{aligned}
P_{i}\left(a_{k}\right) & =\left(1-p_{c}\right) f_{i}\left(a_{k}\right)+p_{c} \frac{1}{q} \\
P_{i j}\left(a_{k}, a_{l}\right) & = \begin{cases}\left(1-p_{c}\right) f_{i j}\left(a_{k}, a_{l}\right)+p_{c} \frac{1}{q^{2}} & \text { for } i \neq j \\
P_{i}\left(a_{k}\right) \delta_{k l} & \text { for } i=j\end{cases}
\end{aligned}
$$

where $p_{c}$ is the ratio of pseudocount. Pseudocount based on Bayesian statistics depends on the number of samples, $B$, and has a characteristic of $p_{c} \rightarrow 0$ for $B \rightarrow \infty ; p_{c}=\kappa /(\kappa+B)$. In the DCA method[71 57], however, $p_{c}=0.5$ was employed independently of the effective number of sequences, $B$.

\subsubsection{Continuous multivariate Gaussian approximation for $P(\sigma)$}

The cross-entropy for the Potts is evaluated by approximating the distribution of sequences with a multivariate Gaussian distribution, $P(\sigma) \simeq \mathcal{N}\left(\left\{\delta_{\sigma_{i} a_{k}}\right\} \mid\left\{P_{i}\left(a_{k}\right)\right\}, \Theta^{-1}\right)$. In this approximation, $q$-gauge is employed, and $P_{i}\left(a_{q}\right), P_{i j}\left(a_{k}, a_{q}\right)=P_{j i}\left(a_{q}, a_{k}\right)$, and $P_{i j}\left(a_{q}, a_{q}\right)$, are regarded as dependent variables; see section 9.2 .1

$$
S_{\text {Potts }}\left(\Theta \mid\left\{P_{i}\right\},\left\{P_{i j}\right\}\right) \simeq-\frac{1}{2}[-\operatorname{Tr} C \Theta+\log \operatorname{det} \Theta-\operatorname{dim} \Theta \log 2 \pi]
$$

where $\Theta$ is a $(q-1) L \times(q-1) L$ precision matrix, which is the inverse covariance matrix, and $C$ is the given covariance matrix defined by Eq. 39

In the case of no regularization term, for which pseudocount (Eqs. 43 and 44 must be employed, the estimate of $\Theta$ is given by

$$
\begin{aligned}
\Theta & =\arg \min _{\Theta} S_{\text {Potts }}\left(\Theta \mid\left\{P_{i}\right\},\left\{P_{i j}\right\}\right)=C^{-1} \\
S_{\text {Potts }}\left(\left\{P_{i}\right\},\left\{P_{i j}\right\}\right) & \simeq \min _{\Theta} S_{\text {Potts }}\left(\Theta \mid\left\{P_{i}\right\},\left\{P_{i j}\right\}\right)=-\left(\log \operatorname{det} C^{-1}\right) / 2+\text { constant }
\end{aligned}
$$

Then, couplings and fields are estimated with Eq. 15 to be

$$
\begin{aligned}
J_{i j}^{\text {Gauss }}\left(a_{k}, a_{l}\right) & =-\frac{\partial S_{\text {Potts }}\left(\left\{P_{i}\right\},\left\{P_{i j}\right\}\right)}{\partial P_{i j}\left(a_{k}, a_{l}\right)}=-\left(C^{-1}\right)_{i j}\left(a_{k}, a_{l}\right) \\
h_{i}^{\text {Gauss }}\left(a_{k}\right) & =-\sum_{j \neq i} \sum_{l \neq q} J_{i j}\left(a_{k}, a_{l}\right) P_{j}\left(a_{l}\right)-\frac{1}{2} \sum_{l \neq q}\left(C^{-1}\right)_{i i}\left(a_{k}, a_{l}\right)\left(\delta_{k l}-2 P_{i}\left(a_{l}\right)\right)
\end{aligned}
$$

Thus, the estimate of $J_{i j}\left(a_{k}, a_{l}\right)$ in the Gaussian approximation without regularization terms is equivalent to that in the mean field approximation. The estimates of fields are different from those (Eq. 36$]$ in the mean field approximation, and give a better generative model[8].

In the following, the $(q-1) L \times(q-1) L$ precision matrix $\Theta$ is estimated by adding regularization terms to the cross entropy of the inverse Potts model. The estimate of $\Theta$ may be employed instead of $C^{-1}$ in Eqs. 48 and 49 to estimate $h$ and $J$. 


\section{With a $\ell_{2}$ regularization term for precision matrix}

In the following case of a $\ell_{2}$ regularization term, the precision matrix $\Theta$ that minimizes the crossentropy defined by Eqs. 8 and 45 can be analytically calculated.

$$
\Theta^{\mathrm{L} 2}=\arg \min _{\Theta}\left[S_{\text {Potts }}\left(\Theta \mid\left\{P_{i}\right\},\left\{P_{i j}\right\}\right)+R(\Theta)\right], R(\Theta) \equiv \frac{\gamma}{2} \sum_{i} \sum_{j} \sum_{k \neq q} \sum_{l \neq q} \Theta_{i j}\left(a_{k}, a_{l}\right)^{2}
$$

The optimum $\Theta\left(=\arg \min _{\Theta} S_{0}\left(\Theta \mid\left\{P_{i}\right\},\left\{P_{i j}\right\}\right)\right)$ is the root of $C-\Theta^{-1}+2 \gamma \Theta=0$. Hence, $\Theta$ and $C$ can be diagonalized by the same orthogonal matrix [16]. Let $\theta_{\lambda}$ and $c_{\lambda}$ denote the $\lambda$ th eigenvalues of $\Theta$ and $C$, respectively. $\theta_{\lambda}$ can be explicitly represented as follows.

$$
\theta_{\lambda}^{-1}=\frac{1}{2}\left(c_{\lambda}+\sqrt{c_{\lambda}^{2}+8 \gamma}\right)
$$

\section{With a $\ell_{1}$ regularization term for precision matrix}

The graphical lasso is employed in PSICOV [45] to infer the precision matrix $\left(C^{-1}\right)_{i j}\left(a_{k}, a_{l}\right)$; the following function, in which a $\ell_{1}$ regularization term corresponding to an exponential prior is employed, is minimized.

$$
\Theta^{\text {glasso }}=\arg \min _{\Theta}\left[S_{\text {Potts }}\left(\Theta \mid\left\{P_{i}\right\},\left\{P_{i j}\right\}\right)+R(\Theta)\right], R(\Theta) \equiv \frac{\gamma}{2} \sum_{i} \sum_{j} \sum_{k \neq q} \sum_{l \neq q}\left|\Theta_{i j}\left(a_{k}, a_{l}\right)\right|
$$

To speed up convergence, a shrinkage method[88] for a sample covariance matrix is also employed in PSICOV [45].

$$
\hat{C}=\lambda C+(1-\lambda) \bar{v} I, \quad \bar{v}=\sum_{i} \sum_{k \neq q} C_{i i}\left(a_{k}, a_{k}\right) /\left(\sum_{i} \sum_{k \neq q} 1\right)
$$

where $0<\lambda<1$ is a parameter, and $I$ is the identity matrix.

In COUSCOus [83], the following shrinkage with an empirical Bayes estimator is performed until the adjusted covariance matrix $\hat{C}$ becomes invertible; $\hat{C}=C+\{(\operatorname{dim} C-1) /(B \operatorname{Tr} C)\} I$. Then, the adjusted covariance matrix $\hat{C}$ is employed instead of $C$ in Eqs. 45 and 52

In PSICOV and COUSCOus, $\Theta$ was supposed to be $q L \times q L$, but in the present model it is $(q-1) L \times(q-1) L$ matrix to consist of linearly independent rows only even in the limit of $\gamma(\propto$ $1 / B) \rightarrow 0$.

\subsubsection{Gaussian approximation for $P(\sigma)$ with a normal-inverse-Wishart prior}

The normal-inverse-Wishart distribution (NIW) is the product of the multivariate normal distribution $(\mathcal{N})$ and the inverse-Wishart distribution $\left(\mathcal{W}^{-1}\right)$, which are the conjugate priors for the mean vector and for the covariance matrix of a multivariate Gaussian distribution, respectively. The NIW is employed as a prior in GaussDCA[7], in which the sequence distribution $P(\sigma)$ is approximated as a Gaussian distribution. In this approximation, the q-gauge is used, and $P_{i}\left(a_{q}\right)$, $P_{i j}\left(a_{k}, a_{q}\right)=P_{j i}\left(a_{q}, a_{k}\right)$, and $P_{i j}\left(a_{q}, a_{q}\right)$ are regarded as dependent variables; see section 9.2.1. in GaussDCA, deletion is excluded from independent variables.

The posterior distribution for the NIW is also a NIW. Thus, the cross entropy $S_{0}$ can be represented as 


$$
\begin{aligned}
S_{0}\left(\mu, \Sigma \mid\left\{P_{i}\right\},\left\{P_{i j}\right\}\right)=\frac{-1}{B} \log \left[\prod_{\tau=1}^{B} \mathcal{N}\left(\left\{\delta_{\sigma_{i}^{\tau} a_{k}}\right\} \mid \mu, \Sigma\right) \mathcal{N}\left(\mu \mid \mu^{0}, \Sigma / \kappa\right) W^{-1}(\Sigma \mid \Lambda, v)\right] \\
=\frac{-1}{B} \log \left[\mathcal{N}\left(\mu \mid \mu^{B}, \Sigma / \kappa^{B}\right) W^{-1}\left(\Sigma \mid \Lambda^{B}, v^{B}\right)\right. \\
\left.\quad(\operatorname{det}(2 \pi \Sigma))^{-B / 2}\left(\frac{\kappa}{\kappa^{B}}\right)^{\operatorname{dim} \Sigma / 2} \frac{(\operatorname{det}(\Lambda / 2))^{v / 2}}{\left(\operatorname{det}\left(\Lambda^{B} / 2\right)\right)^{v^{B} / 2}} \frac{\Gamma_{\operatorname{dim} \Sigma}\left(v^{B} / 2\right)}{\Gamma_{\operatorname{dim} \Sigma}(v / 2)}(\operatorname{det} \Sigma)^{-\left(v-v^{B}\right) 2}\right]
\end{aligned}
$$

where $\Gamma_{\operatorname{dim} \Sigma}(v / 2)$ is the multivariate $\Gamma$ function, $\mu$ is the mean vector, and $\operatorname{dim} \Sigma$ is the dimension of covariance matrix $\Sigma, \operatorname{dim} \Sigma=(q-1) L$ excluding deletion in GaussDCA. The normal and NIW distributions are defined as follows.

$$
\begin{aligned}
\mathcal{N}\left(\mu \mid \mu^{0}, \Sigma\right) & \equiv(\operatorname{det}(2 \pi \Sigma))^{-1 / 2} \exp \left(-\frac{\left(\mu-\mu^{0}\right)^{T} \Sigma^{-1}\left(\mu-\mu^{0}\right)}{2}\right) \\
\mathcal{W}^{-1}(\Sigma \mid \Lambda, \nu) & \equiv \frac{(\operatorname{det}(\Lambda / 2))^{v / 2}}{\Gamma_{\operatorname{dim} \Sigma(v / 2)}(\operatorname{det} \Sigma)^{-(v+\operatorname{dim} \Sigma+1) / 2} \exp \left(-\frac{1}{2} \operatorname{Tr} \Lambda \Sigma^{-1}\right)}
\end{aligned}
$$

Parameters $\mu^{B}, \kappa^{B}, v^{B}$, and $\Lambda^{B}$ satisfy

$$
\begin{aligned}
\mu_{i}^{B}\left(a_{k}\right) & =\left(\kappa \mu_{i}^{0}\left(a_{k}\right)+B P_{i}\left(a_{k}\right)\right) /(\kappa+B), \kappa^{B}=\kappa+B, v^{B}=v+B \\
\Lambda_{i j}^{B}\left(a_{k}, a_{l}\right) & =\Lambda_{i j}\left(a_{k}, a_{l}\right)+B C_{i j}\left(a_{k}, a_{l}\right)+\frac{\kappa B}{\kappa+B}\left[\left(P_{i}\left(a_{k}\right)-\mu_{i}^{0}\left(a_{k}\right)\right)\left(P_{j}\left(a_{l}\right)-\mu_{j}^{0}\left(a_{l}\right)\right)\right]
\end{aligned}
$$

where the $\Lambda$ and $v$ are the scale matrix and the degree of freedom, respectively, shaping the inverseWishart distribution, and $C$ is the given covariance matrix defined by Eq. 39 The mean values of $\mu$ and $\Sigma$ under NW posterior are $\mu^{B}$ and $\Lambda^{B} /\left(v^{B}-\operatorname{dim} \Sigma-1\right)$, and their mode values are $\mu^{B}$ and $\Lambda^{B} /\left(v^{B}+\operatorname{dim} \Sigma+1\right)$, which minimize the cross entropy or maximize the posterior probability. The covariance matrix $\Sigma$ can be estimated to be the exactly same value by adjusting the value of $v$, whichever the mean posterior or the maximum posterior is employed for the estimation of $\Sigma$. In GaussDCA, the mean posterior estimate was employed but here the maximum posterior estimate is employed according to the present formalism.

$$
(\mu, \Sigma)=\arg \min _{(\mu, \Sigma)} S_{0}\left(\mu, \Sigma \mid\left\{P_{i}\right\},\left\{P_{i j}\right\}\right)=\left(\mu^{B}, \Lambda^{B} /\left(v^{B}+\operatorname{dim} \Sigma+1\right)\right)
$$

According to GaussDCA, $v$ is chosen in such a way that $\Sigma_{i j}\left(a_{k}, a_{l}\right)$ is nearly equal to the covariance matrix corrected by pseudocount in Eq. 44. $v=\kappa+\operatorname{dim} \Sigma+1$ for the mean posterior estimate in GaussDCA, but $v=\kappa-\operatorname{dim} \Sigma-1$ for the maximum posterior estimate here.

From Eq. 15 the estimates of couplings and fields are calculated.

$$
J_{i j}^{\mathrm{NIW}}\left(a_{k}, a_{l}\right)=-\frac{\partial S_{0}\left(\left\{P_{i}\right\},\left\{P_{i j}\right\}\right)}{\partial P_{i j}\left(a_{k}, a_{l}\right)}=-\frac{(\kappa+B+1)}{\kappa+B}\left(\Sigma^{-1}\right)_{i j}\left(a_{k}, a_{l}\right)
$$

Because the number of instances is far greater than $1(B \gg 1)$, these estimates of couplings are practically equal to the estimates $\left(J^{\mathrm{MF}}=-\Sigma^{-1}\right)$ in the mean field approximation, which was employed in GaussDCA[7].

$$
\begin{aligned}
h_{i}^{\mathrm{NIW}}\left(a_{k}\right)= & -\sum_{j \neq i} \sum_{l} J_{i j}^{\mathrm{NIW}}\left(a_{k}, a_{l}\right) P_{j}\left(a_{l}\right)-\frac{(\kappa+B+1)}{\kappa+B} \sum_{j} \sum_{l \neq q}\left(\Sigma^{-1}\right)_{i j}\left(a_{k}, a_{l}\right) \\
& {\left[\delta_{i j} \frac{\delta_{k l}-2 P_{i}\left(a_{l}\right)}{2}+\frac{\kappa B}{\kappa+B}\left(P_{j}\left(a_{l}\right)-\mu_{j}^{0}\left(a_{l}\right)\right)\right] }
\end{aligned}
$$

The $\left(h_{i}^{\mathrm{NIW}}\left(a_{k}\right)-h_{i}^{\mathrm{NIW}}\left(a_{q}\right)\right)$ does not converge to $\log P_{i}\left(a_{k}\right) / P_{i}\left(a_{q}\right)$ as $J^{\mathrm{NIW}} \rightarrow 0$ but $h_{i}^{\mathrm{MF}}\left(a_{k}\right)-$ $h_{i}^{\mathrm{MF}}\left(a_{q}\right)$ does; in other words, the mean field approximation gives a better $h$ for the limiting case of 
no couplings than the present approximation. Barton et al.[8] reported that the Gaussian approximation generally gave a better generative model than the mean field approximation.

In GaussDCA[7], $\mu^{0}$ and $\Lambda / \kappa$ were chosen to be as uninformative as possible, i.e., mean and covariance for a uniform distribution.

$$
\mu_{i}^{0}\left(a_{k}\right)=1 / q \quad, \quad \frac{\Lambda_{i j}\left(a_{k}, a_{l}\right)}{\kappa}=\frac{\delta_{i j}}{q}\left(\delta_{k l}-\frac{1}{q}\right)
$$

\subsubsection{Pseudo-likelihood approximation}

\section{Symmetric pseudo-likelihood maximization}

The probability of an instance $\sigma^{\tau}$ is approximated as follows by the product of conditional probabilities of observing $\sigma_{i}^{\tau}$ under the given observations $\sigma_{j \neq i}^{\tau}$ of all other sites.

$$
P\left(\sigma^{\tau}\right) \approx \prod_{i} P\left(\sigma_{i}=\sigma_{i}^{\tau} \mid\left\{\sigma_{j \neq i}=\sigma_{j}^{\tau}\right\}\right)
$$

Then, cross entropy is approximated as

$$
\begin{gathered}
S_{0}\left(h, J \mid\left\{P_{i}\right\},\left\{P_{i j}\right\}\right) \approx S_{0}^{\mathrm{PLM}}\left(h, J \mid\left\{P_{i}\right\},\left\{P_{i j}\right\}\right) \equiv \sum_{i} S_{0, i}\left(h, J \mid\left\{P_{i}\right\},\left\{P_{i j}\right\}\right) \\
S_{0, i}\left(h, J \mid\left\{P_{i}\right\},\left\{P_{i j}\right\}\right) \equiv \frac{-1}{B} \sum_{\tau} \ell_{i}\left(\sigma_{i}=\sigma_{i}^{\tau} \mid\left\{\sigma_{j \neq i}=\sigma_{j}^{\tau}\right\}, h, J\right)+R_{i}(h, J)
\end{gathered}
$$

where conditional log-likelihoods and $\ell_{2}$ norm regularization terms employed in [23] are

$$
\begin{array}{r}
\ell_{i}\left(\sigma_{i}=\sigma_{i}^{\tau} \mid\left\{\sigma_{j \neq i}=\sigma_{j}^{\tau}\right\}, h, J\right)=\log \left[\frac{\exp \left(h_{i}\left(\sigma_{i}^{\tau}\right)+\sum_{j \neq i} J_{i j}\left(\sigma_{i}^{\tau}, \sigma_{j}^{\tau}\right)\right)}{\sum_{k} \exp \left(h_{i}\left(a_{k}\right)+\sum_{j \neq i} J_{i j}\left(a_{k}, \sigma_{j}^{\tau}\right)\right)}\right] \\
R_{i}(h, J) \equiv \gamma_{h} \sum_{k} h_{i}\left(a_{k}\right)^{2}+\frac{\gamma_{J}}{2} \sum_{k} \sum_{j \neq i} \sum_{l} J_{i j}\left(a_{k}, a_{l}\right)^{2}
\end{array}
$$

The optimum fields and couplings in this approximation are estimated by minimizing the pseudocross-entropy, $S_{0}^{\mathrm{PLM}}$.

$$
\left(h^{\mathrm{PLM}}, J^{\mathrm{PLM}}\right)=\arg \min _{h, J} S_{0}^{\mathrm{PLM}}\left(h, J \mid\left\{P_{i}\right\},\left\{P_{i j}\right\}\right)
$$

Eq. 70 is not invariant under gauge transformation; the $\ell_{2}$ norm regularization terms in Eq. 70 favors only a specific gauge that corresponds to $\gamma_{J} \sum_{l} J_{i j}\left(a_{k}, a_{l}\right)=\gamma_{h} h_{i}\left(a_{k}\right), \gamma_{J} \sum_{k} J_{i j}\left(a_{k}, a_{l}\right)=$ $\gamma_{h} h_{j}\left(a_{l}\right)$, and $\sum_{k} h_{i}\left(a_{k}\right)=0$ for all $i, j(>i), k$ and $l[23] . \gamma_{J}=\gamma_{h}=0.01$ that is relatively a large value independent of $B$ was employed in [23]. $\gamma_{h}=0.01$ but $\gamma_{J}=q(L-1) \gamma_{h}$ were employed in [38], in which gapped sites in each sequence were excluded in the calculation of the Hamiltonian $H(\sigma)$, and therefore $q=20$.

GREMLIN [48] employs Gaussian prior probabilities that depend on site pairs.

$$
\begin{aligned}
R_{i}(h, J) & \equiv \gamma_{h} \sum_{k} h_{i}\left(a_{k}\right)^{2}+\sum_{k} \sum_{j \neq i} \frac{\gamma_{i j}}{2} \sum_{l} J_{i j}\left(a_{k}, a_{l}\right)^{2} \\
\gamma_{i j} & \equiv \gamma_{c}\left(1-\gamma_{p} \log \left(P_{i j}^{0}\right)\right)
\end{aligned}
$$

where $P_{i j}^{0}$ is the prior probability of site pair $(i, j)$ being in contact. 


\section{Asymmetric pseudo-likelihood maximization}

To speed up the minimization of $S_{0}$, a further approximation, in which $S_{0, i}$ is separately minimized, is employed[22], and fields and couplings are estimated as follows.

$$
\begin{aligned}
J_{i j}^{\mathrm{PLM}}\left(a_{k}, a_{l}\right) & \simeq \frac{1}{2}\left(J_{i j}^{*}\left(a_{k}, a_{l}\right)+J_{j i}^{*}\left(a_{l}, a_{k}\right)\right) \\
\left(h_{i}^{\mathrm{PLM}}, J_{i}^{*}\right) & =\underset{h_{i}, J_{i}}{\arg \min _{0, i} S_{0}\left(h, J \mid\left\{P_{i}\right\},\left\{P_{i j}\right\}\right)}
\end{aligned}
$$

It is appropriate to transform $h$ and $J$ estimated above into a some specific gauge such as the Ising gauge.

\subsubsection{ACE (adaptive cluster expansion) of cross-entropy for sparse Markov random field}

The cross entropy $S_{0}\left(\left\{h_{i}, J_{i j}\right\} \mid\left\{P_{i}\right\},\left\{P_{i j}\right\}, i, j \in \Gamma\right)$ of a cluster of sites $\Gamma$, which is defined as the negative log-likelihood per instance in Eq. 14 is approximately minimized by taking account of sets $L_{k}(t)$ of only significant clusters consisting of $k$ sites, the incremental entropy (cluster cross entropy) $\Delta S_{\Gamma}$ of which is significant $\left(\left|\Delta S_{\Gamma}\right|>t\right)[15,16,8]$.

$$
\begin{aligned}
S_{0}\left(\left\{P_{i}, P_{i j} \mid i, j \in \Gamma\right\}\right) & \simeq \sum_{l=1}^{|\Gamma|} \sum_{\Gamma^{\prime} \in L_{l}(t), \Gamma^{\prime} \subset \Gamma} \Delta S_{0}\left(\left\{P_{i}, P_{i j} \mid i, j \in \Gamma^{\prime}\right\}\right) \\
\Delta S_{0}\left(\left\{P_{i}, P_{i j} \mid i, j \in \Gamma\right\}\right) & \equiv S_{0}\left(\left\{P_{i}, P_{i j} \mid i, j \in \Gamma\right\}\right)-\sum_{\Gamma^{\prime} \subset \Gamma} \Delta S_{0}\left(\left\{P_{i}, P_{i j} \mid i, j \in \Gamma^{\prime}\right\}\right) \\
& =\sum_{\Gamma^{\prime} \subseteq \Gamma}(-1)^{|\Gamma|-\left|\Gamma^{\prime}\right|} S_{0}\left(\left\{P_{i}, P_{i j} \mid i, j \in \Gamma^{\prime}\right\}\right)
\end{aligned}
$$

$L_{k+1}(t)$ is constructed from $L_{k}(t)$ by adding a cluster $\Gamma$ consisting of $(k+1)$ sites in a lax case provided that any pair of size $k$ clusters $\Gamma^{1}, \Gamma^{2} \in L_{k}(t)$ and $\Gamma^{1} \bigcup \Gamma^{2}=\Gamma$ or in a strict case if $\Gamma^{\prime} \in L_{k}(t)$ for $\forall \Gamma^{\prime}$ such that $\Gamma^{\prime} \subset \Gamma$ and $\left|\Gamma^{\prime}\right|=k$. Thus, Eq. 75 yields sparse solutions. The cross entropies $S_{0}\left(\left\{P_{i}, P_{i j} \mid i, j \in \Gamma^{\prime}\right\}\right)$ for the small size of clusters are estimated by minimizing $S_{0}\left(\left\{h_{i}, J_{i j}\right\} \mid\left\{P_{i}, P_{i j}\right\}, i, j \in \Gamma^{\prime}\right)$ with respect to fields and couplings. Starting from a large value of the threshold $t$ (typically $t=1$ ), the cross-entropy $S_{0}\left(\left\{P_{i}, P_{i j}\right\} \mid i, j \in\{1, \ldots, N\}\right)$ is calculated by gradually decreasing $t$ until its value converges. Convergence of the algorithm may also be more difficult for alignments of long proteins or those with very strong interactions. In such cases, strong regularization may be employed.

The following regularization terms of $\ell_{2}$ norm are employed in ACE[8], and so Eq. 75 is not invariant under gauge transformation.

$$
-\frac{1}{B} \log P_{0}(h, J \mid i, j \in \Gamma)=\gamma_{h} \sum_{i \in \Gamma} \sum_{k} h_{i}\left(a_{k}\right)^{2}+\gamma_{J} \sum_{i \in \Gamma} \sum_{k} \sum_{j>i, j \in \Gamma} \sum_{l} J_{i j}\left(a_{k}, a_{l}\right)^{2}
$$

$\gamma_{h}=\gamma_{J} \propto 1 / B$ was employed[[8].

The compression of the number of Potts states, $q_{i} \leq q$, at each site can be taken into account. All infrequently observed states or states that insignificantly contribute to site entropy can be treated as the same state, and a complete model can be recovered[8] by setting $h_{i}\left(a_{k}\right)=$ $h_{i}\left(a_{k^{\prime}}\right)+\log \left(P_{i}\left(a_{k}\right) / P_{i}^{\prime}\left(a_{k^{\prime}}\right)\right)$, and $J_{i j}\left(a_{k}, a_{l}\right)=J_{i j}^{\prime}\left(a_{k^{\prime}}, a_{l^{\prime}}\right)$, where "," denotes a corresponding aggregated state and a potential.

Starting from the output set of the fields $h_{i}\left(a_{k}\right)$ and couplings $J_{i j}\left(a_{k}, a_{l}\right)$ obtained from the cluster expansion of the cross-entropy, a Boltzmann machine is trained with $P_{i}\left(a_{k}\right)$ and $P_{i j}\left(a_{k}\right)$ by 
the RPROP algorithm [85] to refine the parameter values of $h_{i}$ and $J_{i j}\left(a_{k}, a_{l}\right)$ [8]; see section 9.2.2 This post-processing is also useful because model correlations are calculated.

An appropriate value of the regularization parameter for trypsin inhibitor were much larger $(\gamma=$ 1) for contact prediction than those $\left(\gamma=2 / B=10^{-3}\right)$ for recovering true fields and couplings [8], probably because the task of contact prediction requires the relative ranking of interactions rather than their actual values.

\subsubsection{Scoring methods for contact prediction}

\section{Conditional Mutual Information, $\mathcal{S}_{i j}^{\mathrm{CMI}}$}

Conditional mutual information for each site pair defined as follows is calculated by the Monte Carlo importance sampling, and employed as a score to predict residue-residue contacts [53, 54].

$$
\mathcal{S}_{i j}^{\mathrm{CMI}} \equiv \sum_{\sigma_{m \neq i, j}} \sum_{k} \sum_{l} P\left(\sigma_{i}=a_{k}, \sigma_{j}=a_{l},\left\{\sigma_{m \neq i, j}\right\}\right) \log \frac{P\left(\sigma_{i}=a_{k}, \sigma_{j}=a_{l} \mid\left\{\sigma_{m \neq i, j}\right\}\right)}{P\left(\sigma_{i}=a_{k} \mid\left\{\sigma_{m \neq i, j}\right\}\right) P\left(\sigma_{j}=a_{l} \mid\left\{\sigma_{m \neq i, j}\right\}\right)}(79)
$$

\section{Direct information, $\mathcal{S}_{i j}^{\mathrm{DI}}$}

The message passing DCA (mpDCA) [106] and the mean field DCA (mfDCA) [71, 57] methods employ direct information $\mathcal{S}_{i j}^{\mathrm{DI}}$ defined as follows for scoring; Sander's group renamed this score evolutionary coupling (EC)[37.

$$
\begin{aligned}
\mathcal{S}_{i j}^{\mathrm{EC}} & \equiv \mathcal{S}_{i j}^{\mathrm{DI}} \equiv \sum_{k} \sum_{l} P_{i j}^{\mathrm{DI}}\left(a_{k}, a_{l}\right) \log \frac{P_{i j}^{\mathrm{DI}}\left(a_{k}, a_{l}\right)}{P_{i}\left(a_{k}\right) P_{j}\left(a_{l}\right)} \\
P_{i j}^{\mathrm{DI}}\left(a_{k}, a_{l}\right) & \propto \exp \left(h_{i j}^{\prime}\left(a_{k}\right)+h_{j i}^{\prime}\left(a_{l}\right)+J_{i j}\left(a_{k}, a_{l}\right)\right)
\end{aligned}
$$

where $h_{i j}^{\prime}\left(a_{k}\right)$ and $h_{j i}^{\prime}\left(a_{l}\right)$ are determined to satisfy the following equations in the two-site model.

$$
\sum_{l} P_{i j}^{\mathrm{DI}}\left(a_{k}, a_{l}\right)=\sum_{l} P_{j i}^{\mathrm{DI}}\left(a_{l}, a_{k}\right)=P_{i}\left(a_{k}\right) \quad \text { for } \quad \forall i \text { and } \forall k
$$

A nice characteristic of $S_{i j}^{\mathrm{DI}}$ is its invariance with respect to the gauge freedom of the Potts model, but pseudocount is required to regularize frequencies $P_{i}\left(a_{k}\right)$ [23].

\section{Corrected Frobenius norm ( $L_{22}$ matrix norm), $\mathcal{S}_{i j}^{\mathrm{CFN}}$}

For scoring, plmDCA 23 22] employs the corrected Frobenius norm of $J_{i j}^{\mathrm{I}}$ transformed in the Ising gauge, in which $J_{i j}^{\mathrm{I}}$ does not contain anything that could have been explained by fields $h_{i}$ and $h_{j}$; $J_{i j}^{\mathrm{I}}\left(a_{k}, a_{l}\right) \equiv J_{i j}\left(a_{k}, a_{l}\right)-J_{i j}\left(\cdot, a_{l}\right)-J_{i j}\left(a_{k}, \cdot\right)+J_{i j}(\cdot, \cdot)$ where $J_{i j}\left(\cdot, a_{l}\right)=J_{j i}\left(a_{l}, \cdot\right) \equiv \sum_{k=1}^{q} J_{i j}\left(a_{k}, a_{l}\right) / q$.

$$
\mathcal{S}_{i j}^{\mathrm{CFN}} \equiv \mathcal{S}_{i j}^{\mathrm{FN}}-\mathcal{S}_{. j}^{\mathrm{FN}} \mathcal{S}_{i .}^{\mathrm{FN}} / \mathcal{S}_{. .}^{\mathrm{FN}} \quad, \quad \mathcal{S}_{i j}^{\mathrm{FN}} \equiv \sqrt{\sum_{k \neq \text { gap }} \sum_{l \neq \text { gap }} J_{i j}^{\mathrm{I}}\left(a_{k}, a_{l}\right)^{2}}
$$


where "." denotes average over the indicated variable. This CFN score with the gap state excluded in Eq. 83 performs better[22, 7] than both scores of FN and DI/EC defined in Eq. 80

Corrected $L_{11}$ matrix norm

In PSICOV [45] and COUSCOus [83], the following corrected $L_{11}$ matrix norm is employed.

$$
\mathcal{S}_{i j}^{\mathrm{PSICOV}} \equiv \mathcal{S}_{i j}^{\mathrm{L} 11}-\mathcal{S}_{\cdot j}^{\mathrm{L} 11} \mathcal{S}_{i \cdot}^{\mathrm{L} 11} / \mathcal{S}_{. .}^{\mathrm{L} 11} \quad, \quad \mathcal{S}_{i j}^{\mathrm{L} 11} \equiv \sum_{k} \sum_{l}\left|\Theta_{i j}\left(a_{k}, a_{l}\right)\right|
$$

This type of correction was first employed in [18] in order to reduce entropic and phylogenetic biases. In PSICOV, this corrected score is converted into an estimated positive predictive value (PPV) by fitting a logistic function to the observed distribution of scores 45.

\subsection{Partial correlation of amino acid covariations between sites}

Direct information was defined[101] in the similar form to partial correlation coefficient as

$$
\begin{gathered}
\mathrm{DI}_{i j}^{\mathrm{TS}} \equiv\left(\mathrm{MIr}^{-1}\right)_{i j} / \sqrt{\left(\mathrm{MIr}^{-1}\right)_{i i}\left(\mathrm{MIr}^{-1}\right)_{j j}} \quad, \quad \mathrm{MIr}_{i j} \equiv \mathrm{MI}_{i j} / S_{i j} \\
\mathrm{MI}_{i j}=S_{i}+S_{j}-S_{i j}, S_{i} \equiv-\sum_{k=1}^{q} P_{i}\left(a_{k}\right) \log P_{i}\left(a_{k}\right), S_{i j} \equiv-\sum_{k=1}^{q} \sum_{l=1}^{q} P_{i j}\left(a_{k}\right) \log P_{i j}\left(a_{k}, a_{l}\right)
\end{gathered}
$$

where MI is a mutual information, and MIr is the normalized MI. The pseudocount method of Eq. 44 is employed to make MIr invertible; the ratio of pseudocount $p_{c}=1 /(B+1)$ was employed[101].

\subsection{Partial correlation of amino acid cosubstitutions between sites in protein evolution}

\subsubsection{Mean of characteristic changes accompanied by substitutions at each site in each branch of a phylogenetic tree in a maximum likelihood model}

Amino acid substitutions are approximated to occur independently at each site. Then, if substitutions are assumed to be in the equilibrium state of a time-reversible Markov process, a likelihood $P\left(\mathcal{A}_{i} \mid T, \Theta, \theta_{\alpha}\right)$ of site $i$ in a multiple sequence alignment (MSA) $\mathcal{A}$ in a phylogenetic tree $T$ under a evolutionary model $\Theta$ with a parameter $\theta_{\alpha}$ for the variation of selective constraints 65, 64, 67 can be calculated by taking any node as a root node. Let us assume here that the root node is a left node $\left(v_{b L}\right)$ of a branch $b$.

$$
P\left(\mathcal{A}_{i} \mid T, \Theta, \theta_{\alpha}\right)=\sum_{\kappa} \sum_{\lambda} P\left(\mathcal{A}_{i}, v_{b L}=\kappa, v_{b R}=\lambda \mid T, \Theta, \theta_{\alpha}\right)
$$


where depending on the evolutionary model $\kappa$ and $\lambda$ correspond to the type of codon or amino acid. The likelihood for $v_{b L}=\kappa$ and $v_{b R}=\lambda$ at site $i, P\left(\mathcal{A}_{i}, v_{b L}=\kappa, v_{b R}=\lambda \mid T, \Theta, \theta_{\alpha}\right)$, is calculated as follows [26].

$$
\begin{aligned}
& P\left(\mathcal{A}_{i}, v_{b L}=\kappa, v_{b R}=\lambda \mid T, \Theta, \theta_{\alpha}\right) \equiv \\
& \quad P_{b L}\left(\mathcal{A}_{i} \mid v_{b L}=\kappa, T, \Theta, \theta_{\alpha}\right) f_{\kappa} P\left(\lambda \mid \kappa, t_{b}, \Theta, \theta_{\alpha}\right) P_{b R}\left(\mathcal{A}_{i} \mid v_{b R}=\lambda, T, \Theta, \theta_{\alpha}\right)
\end{aligned}
$$

where $P_{b L}\left(\mathcal{A}_{i} \mid v_{b L}=\kappa, T, \Theta, \theta_{\alpha}\right)$ is a conditional likelihood of the left subtree with $v_{b L}=\kappa$, and $f_{\kappa}$ is the equilibrium frequency of $\kappa, P\left(\left.\lambda\right|_{\kappa}, t_{b}, \Theta, \theta_{\alpha}\right)$ is a substitution probability from $\kappa$ to $\lambda$ at the branch $b$ whose length is equal to $t_{b}$. In the maximum likelihood (ML) method for phylogenetic trees, the tree $T$ and parameters $\Theta$ are estimated by maximizing the likelihood; $(\hat{T}, \hat{\Theta})=\arg \max _{T, \Theta} P(\mathcal{A} \mid T, \Theta)$, where $P(\mathcal{A} \mid T, \Theta)=\sum_{\alpha} \prod_{i} P\left(\mathcal{A}_{i} \mid T, \Theta, \theta_{\alpha}\right) P\left(\theta_{\alpha}\right)$ and $P\left(\theta_{\alpha}\right)$ is a prior probability.

The mean $\Delta_{i b}$ of any quantity $\Delta_{\kappa \lambda}$ accompanied by substitutions from $\kappa$ to $\lambda$ at each site $i$ in each branch $b$ of a phylogenetic tree can be calculated as follows; $\Delta_{\kappa \lambda}$ corresponds to characteristic changes for coevolution such as volume and charge changes due to amino acid substitutions.

$$
\begin{aligned}
\Delta_{i b}\left(\mathcal{A}_{i}, \hat{T}, \hat{\Theta}, \theta_{\alpha}\right) & \equiv \sum_{\kappa, \lambda} \frac{\Delta_{\kappa \lambda} P\left(\mathcal{A}_{i}, v_{b L}=\kappa, v_{b R}=\lambda \mid \hat{T}, \hat{\Theta}, \theta_{\alpha}\right)}{P\left(\mathcal{A}_{i} \mid \hat{T}, \hat{\Theta}, \theta_{\alpha}\right)} \\
\Delta_{i b}\left(\mathcal{A}_{i}, \hat{T}, \hat{\Theta}\right) & =\sum_{\theta_{\alpha}} \Delta_{i b}\left(\mathcal{A}_{i}, \hat{T}, \hat{\Theta}, \theta_{\alpha}\right) P\left(\theta_{\alpha} \mid \mathcal{A}_{i}, \hat{T}, \hat{\Theta}\right)
\end{aligned}
$$

where $P\left(\theta_{\alpha} \mid \mathcal{A}_{i}, \hat{T}, \hat{\Theta}\right)$ is a posterior probability; $P\left(\theta_{\alpha} \mid \mathcal{A}_{i}, \hat{T}, \hat{\Theta}\right)=P\left(\mathcal{A}_{i} \mid \hat{T}, \hat{\Theta}, \theta_{\alpha}\right) P\left(\theta_{\alpha}\right) / P\left(\mathcal{A}_{i} \mid \hat{T}, \hat{\Theta}\right)$. A Bayesian method for mapping mutations on a phylogenetic tree was first discussed by Nielsen[74], and the present formulation of Eqs. 89 and 90 was introduced as a substitution vector along branches at site $i$ by Dutheil et al. [21] for detecting coevolving positions in a molecule. The method named substitution mapping for mapping evolutionary trajectories of discrete traits on phylogenies was further extended [62 63 100], and was shown to provide extremely robust statistics [76, 86].

\subsubsection{Partial correlation coefficients of feature vectors between sites}

If $\Delta_{\kappa \lambda}$ is defined to be equal to 1 for $\kappa \neq \lambda$ and 0 for $\kappa=\lambda, \Delta_{i b}\left(\mathcal{A}_{i}, \hat{T}, \hat{\Theta}\right)$ will represent the expected value of substitution probability at site $i$ in branch $b$. Let us define a vector $\Delta_{i}$ as follows, and consider the correlation of the two vectors, $\Delta_{i}$ and $\Delta_{j}$.

$$
\Delta_{i} \equiv\left(\ldots, \Delta_{i b}\left(\mathcal{A}_{i}, \hat{T}, \hat{\Theta}\right)-\sum_{b} \Delta_{i b}\left(\mathcal{A}_{i}, \hat{T}, \hat{\Theta}\right) / \sum_{b} 1, \ldots\right)^{T}
$$

where $T$ denotes transpose.

The correlation between sites $i$ and $j$ may be an indirect correlation resulting from correlations between sites $i$ and $k$ and between sites $k$ and $j$. To reduce such indirect correlations, partial correlation coefficients are employed here. The partial correlation coefficient is the correlation coefficient between residual vectors $\left(\Pi_{\perp\left\{\Delta_{k \neq i, j}\right\}} \Delta_{i}\right.$ and $\left.\Pi_{\perp\left\{\Delta_{k \neq i, j}\right\}} \Delta_{j}\right)$ of given two vectors that are perpendicular to a subspace consisting of other vectors except those two vectors $\left(\Delta_{i}\right.$ and $\left.\Delta_{j}\right)$ and therefore cannot be accounted for by a linear multiple regression on other vectors; $\Pi_{\perp\left\{\Delta_{k \neq i, j}\right\}}$ is a projection operator to a space perpendicular to the subspace. If the correlation matrix $C$ is invertible, then the partial correlation coefficients $C_{i j}$ will be related to the $(i, j)$ element of its inverse matrix.

$$
C_{i j} \equiv r_{\Pi_{\perp\left\{\Delta_{k \neq i, j}\right\}} \bigcup_{i} \Pi_{\perp\left\{\Delta_{k \neq i, j}\right.} \bigcup_{j}} \equiv \frac{\left(\Pi_{\perp\left\{\Delta_{k \neq i, j}\right\}} \Delta_{i}\right)^{T}\left(\Pi_{\perp\left\{\Delta_{k \neq i, j} \Delta_{j}\right)}\left\|\Pi_{\perp\left\{\Delta_{k \neq i, j}\right\}} \Delta_{i}\right\|\left\|\Pi_{\perp\left\{\Delta_{k \neq i, j}\right\}} \Delta_{j}\right\|\right.}{\sqrt{\left(C^{-1}\right)_{i i}\left(C^{-1}\right)_{j j}}}
$$




\subsubsection{Characteristic variables indicating coevolution between sites}

Characteristic changes accompanied by substitutions whose correlations indicate coevolution between sites have been employed as $\Delta_{\kappa, \lambda}$ in Eq. 89 (1) occurrence of amino acid substitution ( $\left.\Delta_{\kappa, \lambda}^{s} \equiv 1-\delta_{a_{\kappa}, a_{\lambda}}\right)$, (2) changes of side chain volume, (3) side chain charge, (4) hydrogen-bonding capability accompanied by an amino acid substitution, and (5) others [66]. Then, a coevolution score $\rho_{i j}$ based on the partial correlation coefficient is defined for each characteristic. For example, in the case of concurrent substitutions between sites, $\rho_{i j}^{s} \equiv \max \left(C_{i j}^{s}, 0\right)$, because the direct correlation of substitutions must be positive. For volume, charge, and hydrogen-bonding capability changes, $\rho_{i j}^{x} \equiv \max \left(-\operatorname{sgn} C_{i j}^{x}\left(\left|\rho_{i j}^{s} C_{i j}^{x}\right|\right)^{1 / 2}, 0\right)$ with $x=$ volume, charge, or hydrogen-bonding capability. Then, the total score was defined as $\rho_{i j} \equiv \max \left(\rho_{i j}^{s}, \rho_{i j}^{v}, \rho_{i j}^{c}, \rho_{i j}^{h}, \ldots\right)$; refer to [66] for detail.

\section{References}

1. Adhikari, B., Bhattacharya, D., Cao, R., Cheng, J.: CONFOLD: Residue-residue contactguided ab initio protein folding. Proteins 83, 1436-1449 (2015). DOI 10.1002/prot.24829

2. Adhikari, B., Nowotny, J., Bhattacharya, D., Hou, J., Cheng, J.: ConEVA: a toolbox for comprehensive assessment of protein contacts. BMC Bioinformatics 17, 517 (2016). DOI 10.1186/s 12859-016-1404-z

3. Altschuh, D., Vernet, T., Berti, P., Moras, D., Nagai, K.: Coordinated amino acid changes in homologous protein families. Protein Eng. 2, 193-199 (1988)

4. Anishchenko, I., Ovchinnikov, S., Kamisetty, H., Baker, D.: Origins of coevolution between residues distant in protein 3d structures. Proc. Natl. Acad. Sci. USA 114, 9122-9127 (2013). DOI 10.1073/pnas.1702664114

5. Atchley, W.R., Wollenberg, K.R., Fitch, W.M., Terhalle, W., Dress, A.W.: Correlations among amino acid sites in bHLH protein domains: an information theoretic analysis. Mol. Biol. Evol. 17, 164-178 (2000)

6. Balakrishnan, S., Kamisetty, H., Carbonell, J.G., Lee, S.I., Langmead, C.J.: Learning generative models for protein fold families. Proteins 79, 1061-1078 (2011). DOI 10.1002/prot. 22934

7. Baldassi, C., Zamparo, M., Feinauer, C., Procaccini, A., Zecchina, R., Weigt, M., Pagnani, A.: Fast and accurate multivariate Gaussian modeling of protein families: Predicting residue contacts and protein-interaction partners. PLoS ONE 9(3), e92721 (2014). DOI 10.1371/ journal.pone.0092721.

8. Barton, J.P., Leonardis, E.D., Coucke, A., Cocco, S.: ACE: adaptive cluster expansion for maximum entropy graphical model inference. Bioinformatics 32, 3089-3097 (2016). DOI 10.1093/bioinformatics/btw328

9. Braun, W., Go, N.: Calculation of protein conformations by proton-proton distance constraints: A new efficient algorithm. J. Mol. Biol. 186, 611-626 (1985). DOI 10.1016/ 0022-2836(85)90134-2

10. Brünger, A.T.: Version 1.2 of the crystallography and NMR system. Nat. Protoc. 2, 27282733 (2007). DOI 10.1038/nprot.2007.406

11. Burger, L., van Nimwegen, E.: Acurate prediction of protein-protein interactions from sequence alignments using a Bayesian method. Mol. Syst. Biol. 4, 165 (2008)

12. Burger, L., van Nimwegen, E.: Disentangling direct from indirect co-evolution of residues in protein alignments. PLoS Comput. Biol. 6(1), e1000633 (2010). DOI 10.1371/journal.pcbi. 1000633.

13. CASP12: 12th community wide experiment on the critical assessment of techniques of protein structure prediction, http://predictioncenter.org/casp12/ (2017).

14. Cocco, S., Feinauer, C., Figliuzzi, M., Monasson, R., Weigt, M.: Inverse statistical physics of protein sequences: A key issues review. arXiv:1703.01222 [q-bio.BM] (2017) 
15. Cocco, S., Monasson, R.: Adaptive cluster expansion for inferring Boltzmann machines with noisy data. Phys. Rev. Lett. 106, 090601 (2011). DOI 10.1103/PhysRevLett.106.090601

16. Cocco, S., Monasson, R.: Adaptive cluster expansion for the inverse Ising problem: Convergence, algorithm and tests. J. Stat. Phys. 147, 252-314 (2012). DOI 10.1007/ s10955-012-0463-4

17. Doron-Faigenboim, A., Pupko, T.: A combined empirical and mechanistic codon model. Mol. Biol. Evol. 24, 388-397 (2007)

18. Dunn, S.D., Wahl, L.M., Gloor, G.B.: Mutual information without the influence of phylogeny or entropy dramatically improves residue contact prediction. Bioinformatics 24, 333-340 (2008)

19. Dutheil, J.: Detecting coevolving positions in a molecule: why and how to account for phylogeny. Brief. Bioinform. 13, 228-243 (2012)

20. Dutheil, J., Galtier, N.: Detecting groups of coevolving positions in a molecule: a clustering approach. BMC Evol. Biol. 7, 242 (2007)

21. Dutheil, J., Pupko, T., Jean-Marie, A., Galtier, N.: A model-based approach for detecting coevolving positions in a molecule. Mol. Biol. Evol. 22, 1919-1928 (2005)

22. Ekeberg, M., Hartonen, T., Aurell, E.: Fast pseudolikelihood maximization for directcoupling analysis of protein structure from many homologous amino-acid sequences. J. Comput. Phys. 276, 341-356 (2014)

23. Ekeberg, M., Lövkvist, C., Lan, Y., Weigt, M., Aurell, E.: Improved contact prediction in proteins: Using pseudolikelihoods to infer Potts models. Phys. Rev. E 87, 012707 (2013). DOI 10.1103/PhysRevE.87.012707.

24. Fares, M., Travers, S.: A novel method for detecting intramolecular coevolution. Genetics 173, 9-23 (2006)

25. Fariselli, P., Olmea, O., Valencia, A., Casadio, R.: Prediction of contact maps with neural networks and correlated mutations. Protein Eng. 14, 835-843 (2001)

26. Felsenstein, J.: Evolutionary trees from DNA sequences: a maximum likelihood approach. J. Mol. Evol. 17, 368-376 (1981)

27. Finn, R.D., Coggill, P., Eberhardt, R.Y., Eddy, S.R., Mistry, J., Mitchell, A.L., Potter, S.C., Punta, M., Qureshi, M., Sangrador-Vegas, A., Salazar, G.A., Tate, J., Bateman, A.: The Pfam protein families database: towards a more sustainable future. Nucl. Acid Res. 44, D279D285 (2016). DOI 10.1093/nar/gkv1344

28. Fitch, W.M., Markowitz, E.: An improved method for determining codon variability in a gene and its application to the rate of fixation of mutations in evolution. Biochem. Genet. 4, 579-593 (1970)

29. Fleishman, S.J., Yifrach, O., Ben-Tal, N.: An evolutionarily conserved network of amino acids mediates gating in voltage-dependent potassium channels. J. Mol. Biol. 340, 307-318 (2004)

30. Fodor, A.A., Aldrich, R.W.: Influence of conservation on calculations of amino acid covariance in multiple sequence alignment. Proteins 56, 211-221 (2004)

31. Georges, A., Yedidia, J.S.: How to expand around mean-field theory using high-temperature expansions. J. Phys. A: Math. Gen. 24, 2173-2192 (1991)

32. Giraud, B.G., Heumann, J.M., Lapedes, A.S.: Superadditive correlation. Phys. Rev. E 59, 4973-4991 (1999)

33. Göbel, U., Sander, C., Schneider, R., Valencia, A.: Correlated mutations and residue contacts in proteins. Proteins 18, 309-317 (1994)

34. Gulyás-Kovács, A.: Integrated analysis of residue coevolution and protein structure in $\mathrm{ABC}$ transporters. PLoS ONE 7(5), e36546 (2012). DOI 10.1371/journal.pone.0036546.

35. Halabi, N., Rivoire, O., Leibler, S., Ranganathan, R.: Protein sectors: evolutionary units of three-dimensional structure. Cell 138, 774-786 (2009)

36. Havel, T.F., Kuntz, I.D., Crippen, G.M.: The combinatorial distance geometry method for the calculation of molecular conformation. I. A new approach to an old problem. J. Theor. Biol. 104, 359-381 (1983) 
37. Hopf, T.A., Colwell, L.J., Sheridan, R., Rost, B., Sander, C., Marks, D.S.: Three-dimensional structures of membrane proteins from genomic sequencing. Cell 149, 1607-1621 (2012). DOI 10.1016/j.cell.2012.04.012

38. Hopf, T.A., Ingraham, J.B., Poelwijk, F.J., Schärfe, C.P.I., Springer, M., Sander, C., Marks, D.S.: Mutation effects predicted from sequence co-variation. Nature Biotech. 35, 128-135 (2017). DOI 10.1038/nbt.3769

39. Hopf, T.A., Schärfe, C.P.I., Rodrigues, J.P.G.L.M., Green, A.G., Kohlbacher, O., Bonvin, A.M.J.J., Sander, C., Marks, D.S.: Sequence co-evolution gives 3D contacts and structures of protein complexes. eLife 3, e03430 (2014). DOI 10.7554/eLife.03430

40. Ingraham, J., Marks, D.: Variational inference for sparse and undirected models. arXiv:1602.03807 [stat.ML] (2016)

41. Jacquin, H., Gilson, A., Shakhnovich, E., Cocco, S., Monasson, R.: Benchmarking inverse statistical approaches for protein structure and design with exactly solvable models. PLoS Comput. Biol. 12, e1004889 (2016). DOI 10.1371/journal.pcbi.1004889

42. Johnson, L.S., Eddy, S.R., Portugaly, E.: Hidden Markov model speed heuristic and iterative HMM search procedure. BMC Bioinformatics 11, 431 (2010)

43. Jones, D.T.: Predicting novel protein folds by using FRAGFOLD. Proteins 45(S5), 127-132 (2001)

44. Jones, D.T., Bryson, K., Coleman, A., McGuffin, L.J., Sadowski, M.I., Sodhi, J.S., Ward, J.J.: Prediction of novel and analogous folds using fragment assembly and fold recognition. Proteins 61(S7), 143-151 (2005). DOI 10.1002/prot.20731

45. Jones, D.T., Buchan, D.W.A., Cozzetto, D., Pontil, M.: PSICOV: precise structural contact prediction using sparse inverse covariance estimation on large multiple sequence alignments. Bioinformatics 28, 184-190 (2012). DOI 10.1093/bioinformatics/btr638

46. Jones, D.T., Singh, T., Kosciolek, T., Tetchner, S.: MetaPSICOV: combining coevolution methods for accurate prediction of contacts and long range hydrogen bonding in proteins. Bioinformatics 31, 999-1006 (2015). DOI 10.1093/bioinformatics/btu791

47. Kaján, L., Hopf, T.A., Kalaš, M., Marks, D.S., Rost, B.: FreeContact: fast and free software for protein contact prediction from residue co-evolution. BMC Bioinformatics 15, 85 (2014)

48. Kamisetty, H., Ovchinnikov, S., Baker, D.: Assessing the utility of coevolution-based residue-residue contact predictions in a sequence- and structure-rich era. Proc. Natl. Acad. Sci. USA 110, 15,674-15,679 (2013). DOI 10.1073/pnas.1314045110

49. Kim, D.E., Blum, B., Bradley, P., Baker, D.: Sampling bottlenecks in de novo protein structure prediction. J. Mol. Biol. 393, 249-260 (2009)

50. Kim, D.E., Chivian, D., Baker, D.: Protein structure prediction and analysis using the Rosetta server. Nucl. Acid Res. 32, W526-W531 (2004)

51. Kosciolek, T., Jones, D.T.: De novo structure prediction of globular proteins aided by sequence variation-derived contacts. PLoS ONE 9, e92197 (2014). DOI 10.1371/journal.pone. 0092197

52. Kosciolek, T., Jones, D.T.: Accurate contact predictions using covariation techniques and machine learning. Proteins 84(S1), 145-151 (2016). DOI 10.1002/prot.24863

53. Lapedes, A., Giraud, B., Jarzynsk, C.: Using sequence alignments to predict protein structure and stability with high accuracy. LANL Sciece Magagine LA-UR-02-4481 (2002)

54. Lapedes, A., Giraud, B., Jarzynsk, C.: Using sequence alignments to predict protein structure and stability with high accuracy. arXiv:1207.2484 [q-bio.QM] (2012)

55. Lapedes, A.S., Giraud, B.G., Liu, L.C., Stormo, G.D.: Correlated mutations in protein sequences: phylogenetic and structural effects. In: F. Seillier-Moiseiwitsch (ed.) IMS Lecture Notes: Statistics in Molecular Biology and Genetics: Selected Proceedings of the Joint AMSIMS-SIAM Summer Conference on Statistics in Molecular Biology, June 22-26, 1997, pp. 345-352. Institute of Mathematical Statistics (1999)

56. Maisnier-Patin, S., Andersson, D.I.: Adaptation to the deleterious effect of antimicrobial drug resistance mutations by compensatory evolution. Res. Microbiol. 155, 360-369 (2004)

57. Marks, D.S., Colwell, L.J., Sheridan, R., Hopf, T.A., Pagnani, A., Zecchina, R., Sander, C.: Protein 3D structure computed from evolutionary sequence variation. PLoS ONE 6(12), e28766 (2011). DOI 10.1371/journal.pone.0028766. 
58. Marks, D.S., Hopf, T.A., Sander, C.: Protein structure prediction from sequence variation. Nature Biotech. 30, 1072-1080 (2012). DOI 10.1038/nbt.2419

59. Martin, L.C., Gloor, G.B., Dunn, S.D., Wahl, L.M.: Using information theory to search for co-evolving residues in proteins. Bioinformatics 21, 4116-4124 (2005)

60. Metropolis, N., Rosenbluth, A.W., Rosenbluth, M.N., Teller, A.H., Teller, E.: Equation of state calculations by fast computing machines. J. Chem. Phys. 21, 1087-1092 (1953)

61. Mézard, M., Mora, T.: Constraint satisfaction and neural networks: A statisticalphysics perspective. arXiv:0803.3061 [q-bio.NC] (2008)

62. Minin, V.N., Suchard, M.A.: Counting labeled transitions in continuous-time Markov models of evolution. J. Math. Biol. 56, 391-412 (2008)

63. Minin, V.N., Suchard, M.A.: Fast, accurate and simulation-free stochastic mapping. Philos. Trans. R. Soc. Lond. B Biol. Sci. 363, 3985-3995 (2008)

64. Miyazawa, S.: Advantages of a mechanistic codon substitution model for evolutionary analysis of protein-coding sequences. PLoS ONE 6(12), e28892 (2011). DOI 10.1371/journal. pone.0028892.

65. Miyazawa, S.: Selective constraints on amino acids estimated by a mechanistic codon substitution model with multiple nucleotide changes. PLoS ONE 6(3), e17244 (2011). DOI 10.1371/journal.pone.0017244.

66. Miyazawa, S.: Prediction of contact residue pairs based on co-substitution between sites in protein structures. PLoS ONE 8(1), e54252 (2013). DOI 10.1371/journal.pone.0054252.

67. Miyazawa, S.: Superiority of a mechanistic codon substitution model even for protein sequences in phylogenetic analysis. BMC Evol. Biol. 13, 257 (2013). DOI 10.1186/ 1471-2148-13-257

68. Miyazawa, S.: Selection originating from protein stability/foldability: Relationships between protein folding free energy, sequence ensemble, and fitness. J. Theor. Biol. 433, 21-38 (2017). DOI 10.1016/j.jtbi.2017.08.018

69. Miyazawa, S.: Prediction of structures and interactions from genome information. In: H. Nakamura (ed.) Integrative Structural Biology with Hybrid Methods, Advances in Experimental Medicine and Biology 1105, chap. 9. Springer Nature Singapore Pte Ltd. (2018). DOI 10.1007/978-981-13-2200-6_9

70. Miyazawa, S., Jernigan, R.L.: Residue-residue potentials with a favorable contact pair term and an unfavorable high packing density term for simulation and threading. J. Mol. Biol. 256, 623-644 (1996). DOI 10.1006/jmbi.1996.0114

71. Morcos, F., Pagnani, A., Lunt, B., Bertolino, A., Marks, D.S., Sander, C., Zecchina, R., Onuchic, J.N., Hwa, T., Weigt, M.: Direct-coupling analysis of residue coevolution captures native contacts across many protein families. Proc. Natl. Acad. Sci. USA 108, E1293-E1301 (2011). DOI 10.1073/pnas.1111471108

72. Morcos, F., Schafer, N.P., Cheng, R.R., Onuchic, J.N., Wolynes, P.G.: Coevolutionary information, protein folding landscapes, and the thermodynamics of natural selection. Proc. Natl. Acad. Sci. USA 111, 12,408-12,413 (2014). DOI 10.1073/pnas.1413575111

73. Moult, J., Fidelis, K., Kryshtafovych, A., Schwede, T., Tramontano, A.: Critical assessment of methods of protein structure prediction: Progress and new directions in round XI. Proteins 84(S1), 4-14 (2016). DOI 10.1002/prot.25064

74. Nielsen, R.: Mapping mutations on phylogenies. Syst. Biol. 51, 729-739 (2002)

75. Nugent, T., Jones, D.T.: Accurate de novo structure prediction of large transmembrane protein domains using fragmentassembly and correlated mutation analysis. Proc. Natl. Acad. Sci. USA 109, E1540-E1547 (2012). DOI 10.1073/pnas.1120036109

76. ÓBrien, J.D., Minin, V.N., Suchard, M.A.: Learning to count: robust estimates for labeled distances between molecular sequences. Mol. Biol. Evol. 26, 801-814 (2009)

77. Ovchinnikov, S., Kim, D.E., Wang, R.Y.R., Liu, Y., DiMaio, F., Baker, D.: Improved de novo structure prediction in CASP11 by incorporating coevolution information into Rosetta. Proteins 84(S1), 67-75 (2016). DOI 10.1002/prot.24974

78. Pazos, F., Helmer-Citterich, M., Ausiello, G., Valencia, A.: Correlated mutations contain information about protein-protein interaction. J. Mol. Biol. 271, 511-523 (1997) 
79. Plefka, T.: Convergence condition of the TAP equation for the infinite-ranged ising spin glass model. J. Phys. A: Math. Gen. 15, 1971-1978 (1982)

80. Pollock, D.D., Taylor, W.R.: Effectiveness of correlation analysis in identifying protein residues undergoing correlated evolution. Protein Eng. 10, 647-657 (1997)

81. Pollock, D.D., Taylor, W.R., Goldman, N.: Coevolving protein residues: maximum likelihood identification and relationship to structure. J. Mol. Biol. 287, 187-198 (1999)

82. Poon, A.F.Y., Lewis, F.I., Frost, S.D.W., Kosakovsky Pond, S.L.: Spidermonkey: rapid detection of co-evolving sites using Bayesian graphical models. Bioinformatics 24, 1949-1950 (2008)

83. Rawi, R., Mall, R., Kunji, K., Anbari, M.E., Aupetit, M., Ullah, E., Bensmail, H.: COUSCOus: improved protein contact prediction using an empirical bayes covariance estimator. BMC Bioinformatics 17, 533 (2016). DOI 10.1186/s12859-016-1400-3

84. Remmert, M., Biegert, A., Hauser, A., Söding, J.: HHblits: lightning-fast iterative protein sequence searching by HMM-HMM alignment. Nature Methods 9, 173-175 (2012)

85. Riedmiller, M., Braun, H.: A direct adaptive method for faster backpropagation learning: the RPROP algorithm. IEEE International Conference on Neural Networks pp. 586-591 (1993)

86. Romiguier, J., Figuet, E., Galtier, N., Douzery, E.J., Boussau, B., Dutheil, J.Y., Ranwez, V.: Fast and robust characterization of time-heterogeneous sequence evolutionary processes using substitution mapping. PLoS ONE 7, e33852 (2012)

87. Russ, W.P., Lowery, D.M., Mishra, P., Yaffe, M.B., Ranganathan, R.: Natural-like function in artificial WW domains. Nature 437, 579-583 (2005)

88. Schäfer, J., Strimmer, K.: A shrinkage approach to large-scale covariance matrix estimation and implications for functional genomics. Stat. Appl. Genet. Mol. Biol. 4, Article 32 (2005)

89. Seemayer, S., Gruber, M., Söding, J.: CCMpred-fast and precise prediction of protein residue-residue contacts from correlated mutations. Bioinformatics 30, 3128-3130 (2014). DOI 10.1093/bioinformatics/btu500

90. Sfriso, P., Duran-Frigola, M., Mosca, R., Emperador, A., Aloy, P., Orozco, M.: Residues coevolution guides the systematic identification of alternative functional conformations in proteins. Structure 24, 116-126 (2016). DOI 10.1016/j.str.2015.10.025

91. Shendure, J., Ji, H.: EPSILON-CP: using deep learning to combine information from multiple sources for protein contact prediction. BMC Bioinformatics 18, 303 (2017). DOI 10.1186/s12859-017-1713-x

92. Shindyalov, I.N., Kolchanov, N.A., Sander, C.: Can three-dimensional contacts in protein structures be predicted by analysis of correlated mutations? Protein Eng. 7, 349-358 (1994)

93. Skerker, J.M., Perchuk, B.S., Siryaporn, A., Lubin, E.A., Ashenberg, O., Goulian, M., Laub, M.T.: Rewiring the specificity of two-component signal transduction systems. Cell 133, 1043-1054 (2008)

94. Skwark, M.J., Abdel-Rehim, A., Elofsson, A.: PconsC: combination of direct information methods and alignments improves contact prediction. Bioinformatics 29, 1815-1816 (2013)

95. Skwark, M.J., Michel, M., Hurtado, D.M., Ekeberg, M., Elofsson, A.: Accurate contact predictions for thousands of protein families using PconsC3. bioRXiv (2016). DOI 10.1101/079673

96. Skwark, M.J., Raimondi, D., Michel, M., Elofsson, A.: Improved contact predictions using the recognition of protein like contact patterns. PLoS Comput. Biol. 10, e1003889 (2014). DOI 10.1371/journal.pcbi.1003889

97. Sułkowska, J.I., Morcos, F., Weigt, M., Hwa, T., Onuchic, J.N.: Genomics-aided structure prediction. Proc. Natl. Acad. Sci. USA 109, 10,340-10,345 (2012). DOI 10.1073/pnas. 1207864109

98. Sutto, L., Marsili, S., Valencia, A., Gervasio, F.L.: From residue coevolution to protein conformational ensembles and functional dynamics. Proc. Natl. Acad. Sci. USA 112, 13,56713,572 (2015). DOI 10.1073/pnas.1508584112

99. Talavera, D., Lovell, S.C., Whelan, S.: Covariation is a poor measure of molecular coevolution. Mol. Biol. Evol. 32, 2456-2468 (2015). DOI 10.1093/molbev/msv109

100. Tataru, P., Hobolth, A.: Comparison of methods for calculating conditional expectations of sufficient statistics for continuous time Markov chains. BMC Bioinformatics 12, 465 (2011) 
101. Taylor, W.R., Sadowski, M.I.: Structural constraints on the covariance matrix derived from multiple aligned protein sequences. PLoS ONE 6(12), e28265 (2011). DOI 10.1371/journal. pone.0028265.

102. Tokuriki, N., Tawfik, D.S.: Protein dynamism and evolvability. Science 324, 203-207 (2009)

103. Toth-Petroczy, A., Palmedo, P., Ingraham, J., Hopf, T.A., Berger, B., Sander, C., Marks, D.S.: Structured states of disordered proteins from genomic sequences. Cell 167, 158-170 (2016). DOI 10.1016/j.cell.2016.09.010

104. Tufféry, P., Darlu, P.: Exploring a phylogenetic approach for the detection of correlated substitutions in proteins. Mol. Biol. Evol. 17, 1753-1759 (2000)

105. Wang, S., Sun, S., Li, Z., Zhang, R., Xu, J.: Accurate de novo prediction of protein contact map by ultra-deep learning model. PLoS Comput. Biol. 13, e1004324 (2017). DOI 10.1371/ journal.pcbi.1005324

106. Weigt, M., White, R.A., Szurmant, H., Hoch, J.A., Hwa, T.: Identification of direct residue contacts in protein-protein interaction by message passing. Proc. Natl. Acad. Sci. USA 106, 67-72 (2009). DOI 10.1073/pnas.0805923106

107. Weinreb, C., Riesselman, A.J., Ingraham, J.B., Gross, T., Sander, C., Marks, D.S.: 3D RNA and functional interactions from evolutionary couplings. Cell 165, 1-13 (2016). DOI 10. 1016/j.cell.2016.03.030

108. Wuyun, Q., Zheng, W., Peng, Z., Yang, J.: A large-scale comparative assessment of methods for residue-residue contact prediction. Brief. Bioinform. 19, 219-230 (2016). DOI 10.1093/ bib/bbw106

109. Yanovsky, C., Horn, V., Thorpe, D.: Protein structure relationships revealed by mutation analysis. Science 146, 1593-1594 (1964) 